\title{
Tuning the GENIE pion production model with MINERvA data
}

P. Stowell, ${ }^{1}$ L. Pickering, ${ }^{2,3}$ C. Wilkinson,${ }^{4}$ C. Wret,${ }^{5,3}$ F. Akbar,${ }^{6}$ D. A. Andrade, ${ }^{7}$ M. V. Ascencio, ${ }^{8}$ L. Bellantoni, ${ }^{9}$ A. Bercellie, ${ }^{5}$ M. Betancourt, ${ }^{9}$ A. Bodek,${ }^{5}$ A. Bravar, ${ }^{10}$ H. Budd ${ }^{5}$ G. Caceres, ${ }^{11}$ T. Cai, ${ }^{5}$ M. F. Carneiro, ${ }^{12}$ J. Chaves, ${ }^{13}$ H. da Motta, ${ }^{11}$ S. A. Dytman, ${ }^{14}$ G. A. Díaz ${ }^{5,8}$ J. Felix,${ }^{7}$ L. Fields, ${ }^{9,15}$ A. Filkins, ${ }^{16}$ R. Fine,${ }^{5}$ N. Fiza, ${ }^{17}$ R. Galindo, ${ }^{18}$ H. Gallagher, ${ }^{19}$ A. Ghosh ${ }^{18,11}$ R. Gran, ${ }^{20}$ D. A. Harris, ${ }^{9}$ S. Henry, ${ }^{5}$ S. Jena, ${ }^{17}$ D. Jena, ${ }^{9}$ J. Kleykamp, ${ }^{5}$ M. Kordosky, ${ }^{16}$ D. Last, ${ }^{13}$ T. Le, ${ }^{19,21}$ X.-G. Lu, ${ }^{22}$ E. Maher, ${ }^{23}$ S. Manly, ${ }^{5}$ W. A. Mann ${ }^{19}$ C. M. Marshall, ${ }^{5,}$ K. S. McFarland, ${ }^{5,9}$ B. Messerly, ${ }^{14}$ J. Miller, ${ }^{18}$ J. G. Morfín, ${ }^{9}$ J. Mousseau, ${ }^{24, \dagger}$ D. Naples, ${ }^{14}$ J. K. Nelson, ${ }^{16}$ C. Nguyen, ${ }^{24}$ A. Norrick, ${ }^{16}$ Nuruzzaman, ${ }^{21,18}$ V. Paolone, ${ }^{14}$ G. N. Perdue,${ }^{9,5}$ M. A. Ramírez, ${ }^{7}$ R. D. Ransome, ${ }^{21}$ H. Ray, ${ }^{24}$ D. Rimal, ${ }^{24}$

P. A. Rodrigues, ${ }^{25,5}$ D. Ruterbories, ${ }^{5}$ H. Schellman, ${ }^{12,15}$ C. J. Solano Salinas, ${ }^{26}$ H. Su, ${ }^{14}$ M. Sultana, ${ }^{5}$ V. S. Syrotenko, ${ }^{19}$ E. Valencia, ${ }^{16,7}$ J. Wolcott, ${ }^{5, \$}$ M. Wospakrik, ${ }^{24}$ B. Yaeggy, ${ }^{18}$ L. Zazueta, ${ }^{16}$ and D. Zhang ${ }^{16}$

(MINERvA Collaboration)

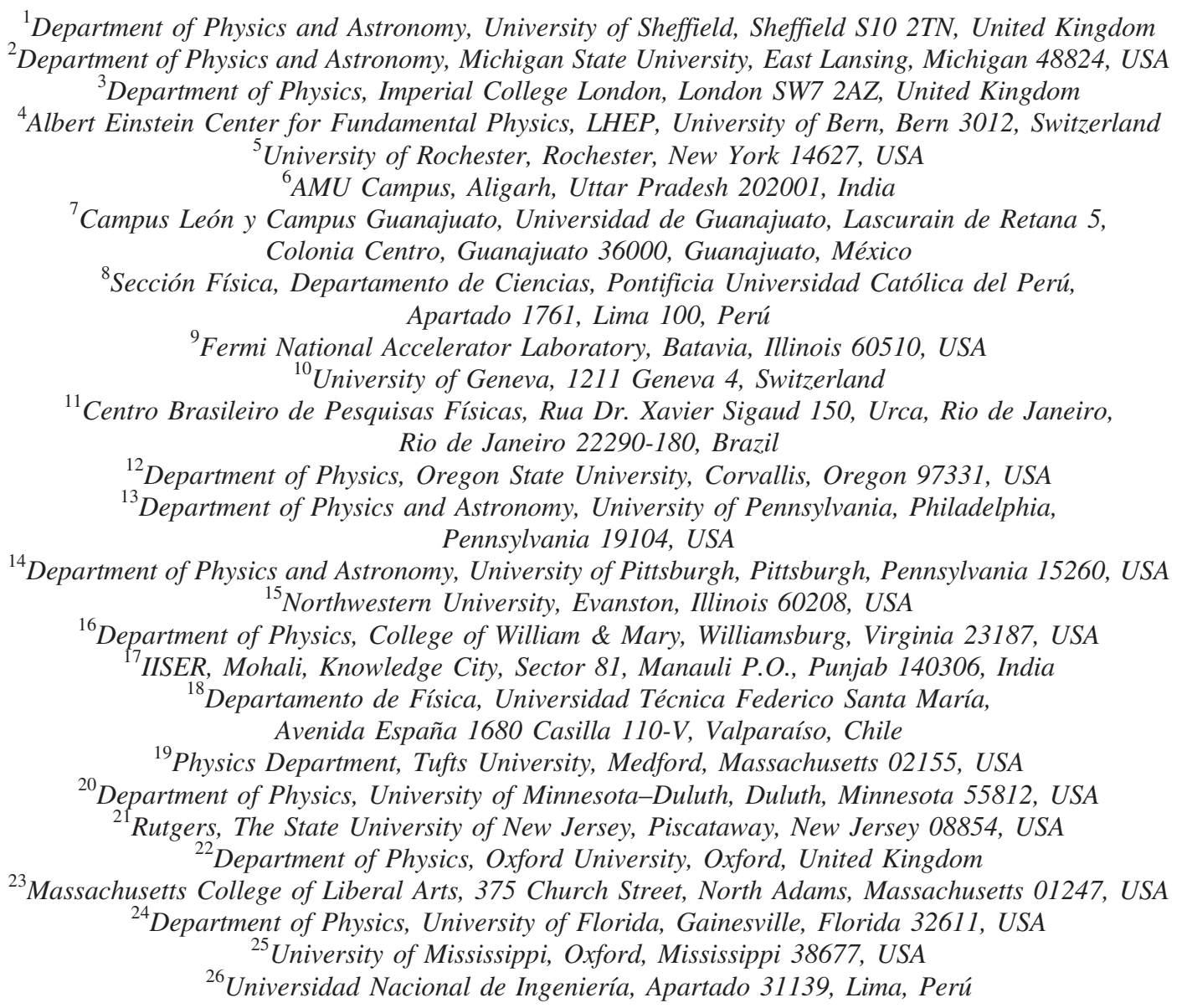

(Received 12 March 2019; published 14 October 2019)

\footnotetext{
*Present address: Lawrence Berkeley National Laboratory, Berkeley, California 94720, USA.

†Present address: University of Michigan, Ann Arbor, Michigan 48109, USA.

${ }^{*}$ Present address: Tufts University, Medford, Massachusetts 02155, USA.
}

Published by the American Physical Society under the terms of the Creative Commons Attribution 4.0 International license. Further distribution of this work must maintain attribution to the author(s) and the published article's title, journal citation, and DOI. Funded by $S C O A P^{3}$. 
Faced with unresolved tensions between neutrino interaction measurements at few-GeV neutrino energies, current experiments are forced to accept large systematic uncertainties to cover discrepancies between their data and model predictions. The widely used pion production model in GENIE is compared to four MINER $\nu \mathrm{A}$ charged current pion production measurements using NUISANCE. Tunings, i.e., adjustments of model parameters, to help match GENIE to MINER $\nu \mathrm{A}$ and older bubble chamber data are presented. We find that scattering off nuclear targets as measured in MINER $\nu \mathrm{A}$ is not in good agreement with expectations based upon scattering off nucleon (hydrogen or deuterium) targets in existing bubble chamber data. An additional ad hoc correction for the low- $Q^{2}$ region, where collective nuclear effects are expected to be large, is presented. While these tunings and corrections improve the agreement of GENIE with the data, the modeling is imperfect. The development of these tunings within the NUISANCE framework allows for straightforward extensions to other neutrino event generators and models, and allows omitting and including new datasets as they become available.

DOI: 10.1103/PhysRevD.100.072005

\section{INTRODUCTION}

In recent years, experimental groups have started to publish neutrino interaction cross-section measurements on nuclear targets in terms of measurable final state particle content, instead of inferred initial interaction channels. This avoids the problem of correcting for complex nuclear effects to make a measurement in terms of the initial interaction channels. For example, events with only a single pion can be produced by the decay of hadronic resonances formed at the primary neutrino interaction, followed by loss of a nucleon from the resonance's decay as a result of final state interactions (FSIs) within the nuclear medium. Such events can also be produced by other sequences of interactions, such as a deep inelastic collision where only a single pion is produced after FSIs. A measurement of charged current events with one identified pion in the final state is a benchmark for models, independent of the details of how each model assesses any particular interaction channel's contributions to that final state. The limitation of giving results in terms of final state particle content is that FSIs are important, and result in the contribution of many different interaction channels into a specific final state.

There are tensions between published data from the $\mathrm{T} 2 \mathrm{~K}$, MiniBooNE Collaboration, and MINER $\nu \mathrm{A}$ experiments [1-5]. These tensions exist in the charged current production of both zero and one pion final states, and a model has yet to emerge that can reliably simulate all experiments at once. This is troubling, as current and future neutrino oscillation experiments require a cross-section model which is predictive across the range of energies covered by these experiments and for a variety of targets.

The differences in neutrino fluxes, scattering targets, available phase space, and signal definitions between experiments make it difficult to diagnose the exact causes of disagreement within the global dataset. In particular, as results must be averaged over the neutrino flux distribution of each experiment, it is difficult to disentangle the energy dependence of an observed deficiency in a particular model, and to decide how uncertainties should be propagated in neutrino energy. Tensions between measurements from a single experiment can uncover fundamental problems with a model which should be addressed, before considering the more difficult issue of developing, or empirically tuning, a model which fits data from multiple experiments. In this work, we employ published MINER $\nu \mathrm{A}$ pion production data. The crosssection measurements utilized in this effort have not been reanalyzed or modified in any way.

NUISANCE [6] was developed to provide the neutrino scattering community with a flexible framework in which various neutrino interaction generators can be validated and empirically tuned to data. Its structure allows for generator tunings to be easily adapted to account for changes in the underlying model or data. In this work, the default pion production model in the GENIE [7,8] neutrino interaction generator is tuned to MINER $\nu \mathrm{A}$ data. Although more sophisticated pion production models exist (e.g., [9-12]), GENIE is widely used by the neutrino scattering community, and its model uncertainties have a central importance to the field. Although the work is only directly applicable to one generator, the methods developed in this paper are easily adaptable to different generators. All the data and methods are publicly available and integrated into the open source NUISANCE framework, facilitating similar studies using other generators and models.

In Sec. II, the data are reviewed and the goodness-of-fit test statistic is defined for the tuning process. Section III describes the default GENIE pion production model, and reviews comparisons of this model to data. In Sec. IV, the parameter reweighting package in GENIE is discussed along with the specific parameters tuned therewith. We also discuss other corrections to the GENIE model made to improve agreement with bubble chamber data $[13,14]$. In Sec. V, we tune additional systematic parameters in GENIE to improve agreement with the MINER $\nu \mathrm{A}$ data in combination with the bubble chamber data. In Sec. VI, additional low- $Q^{2}$ ad hoc corrections are added to the model to 
TABLE I. Summary of the measurements used in this analysis. $W_{\text {rec }}$ is the true reconstructed hadronic mass assuming the struck nucleon is at rest. None of the measurements veto on activity other than the $\mu$ and $\pi$ in their signal definition, and all selections require $1.5<E_{\nu}<20 \mathrm{GeV}$.

\begin{tabular}{lcccc}
\hline \hline Channel & $\nu_{\mu} \mathrm{CC} 1 \pi^{ \pm}[17]$ & $\nu_{\mu} \mathrm{CCN} \pi^{ \pm}[18]$ & $\nu_{\mu} \mathrm{CC} 1 \pi^{0}[19]$ & $\bar{\nu}_{\mu} \mathrm{CC} 1 \pi^{0}[18]$ \\
\hline$N_{\text {bins }} p_{\mu}$ & 8 & 9 & 8 & 9 \\
$N_{\text {bins }} \theta_{\mu}$ & 9 & 9 & 9 & 9 \\
$N_{\text {bins }} T_{\pi}$ & 7 & 7 & 7 & 7 \\
$N_{\text {bins }} \theta_{\pi}$ & 14 & 14 & 11 & 11 \\
$N_{\text {bins }}$ total & 38 & 39 & 35 & 36 \\
Signal definition & $1 \pi^{ \pm}, \geq 0 \pi^{0}$ & $>0 \pi^{ \pm}, \geq 0 \pi^{0}$ & $1 \pi^{0}, 0 \pi^{ \pm}$ & $1 \pi^{0}, 0 \pi^{ \pm}$ \\
& $1 \mu^{-}$ & $1 \mu^{-}$ & $1 \mu^{-}$ & $1 \mu^{+}$ \\
& $W_{\text {rec }}<1.4 \mathrm{GeV}$ & $W_{\text {rec }}<1.8 \mathrm{GeV}$ & $W_{\text {rec }}<1.8 \mathrm{GeV}$ & $W_{\text {rec }}<1.8 \mathrm{GeV}$ \\
& not applicable & not applicable & $\theta_{\mu}<25^{\circ}$ & not applicable \\
\hline \hline
\end{tabular}

resolve observed tensions, motivated by the need for similar corrections observed at both MINOS [15] and MiniBooNE [16]. Finally, in Sec. VII we present our conclusions.

\section{DATA INCLUDED IN THE FITS}

We tune to four of MINER $\nu$ A's published charged current pion production measurements taken on a polystyrene scintillator target: $\nu_{\mu} \mathrm{CC} 1 \pi^{ \pm}$[17], $\nu_{\mu} \mathrm{CCN} \pi^{ \pm}$[18], $\nu_{\mu} \mathrm{CC} 1 \pi^{0}[19]$, and $\bar{\nu}_{\mu} \mathrm{CC} 1 \pi^{0}[18]$, summarized in Table I. ${ }^{1}$ The MINER $\nu$ A detector [20] does not determine the polarity of charged pions. The fraction of $\pi^{-}$in the $\nu_{\mu} \mathrm{CC} 1 \pi^{ \pm}$sample is small $(\sim 1 \%)$. Furthermore, the $\nu_{\mu} \mathrm{CC} 1 \pi^{ \pm}$and $\nu_{\mu} \mathrm{CCN} \pi^{ \pm}$ signal definition allows for any number of neutral pions. Approximately $3 \%$ of the MINER $\nu \mathrm{A} \nu_{\mu} \mathrm{CC} 1 \pi^{ \pm}$signal events have at least one neutral pion in the final state. All four analyses include signal definition cuts on the true "reconstructed" mass of the hadronic system assuming the struck nucleon is at rest, $W_{\text {rec }}$, and the true neutrino energy $E_{\nu}$.

The kinematic variable distributions used in this work are the momentum and angle of the outgoing muon with respect to the incoming neutrino beam, $p_{\mu}$ and $\theta_{\mu}$, and the kinetic energy and angle of the outgoing pion with respect to the incoming neutrino beam, $T_{\pi}$ and $\theta_{\pi}$. In the $\nu_{\mu} \mathrm{CCN} \pi^{ \pm}$ channel, where there is at least one $\pi^{ \pm}$in the final state, there is one entry in the distributions of $\theta_{\pi}$ and $T_{\pi}$ for each $\pi^{ \pm}$in an event. The data are reported as efficiency corrected results unfolded to true kinematic variables, which may introduce model dependence. This is notably problematic in regions of low efficiency-present in the charged pion channels at $\theta_{\pi} \sim 90^{\circ}, T_{\pi}<50 \mathrm{MeV}$, and $T_{\pi}>350 \mathrm{MeV}$, where the signal efficiency is zero [17]. The pion selection cuts, not present in the signal definition, remove about $50 \%$ of the signal events, with little dependence upon the muon variables, but a clear impact on the shape of the pion kinematic variables.

\footnotetext{
${ }^{1}$ In " $\nu_{\mu} \mathrm{CCN} \pi^{ \pm}$," the $N$ indicates one or more identified pions and does not refer to a nucleon.
}

The published cross sections are one dimensional with correlations provided between the bins within each distribution. No correlations are provided between measurements of different final states, or between different onedimensional projections of the same measurement. These correlations are expected to be large, coming predominantly from flux and detector uncertainties. Additionally, the $\nu_{\mu} \mathrm{CC} 1 \pi^{ \pm}$event sample is a subset $(\sim 64 \%)$ of the $\nu_{\mu} \mathrm{CCN} \pi^{ \pm}$event sample, and including both channels introduces a statistical correlation. Not assessing correlations between the distributions, while a common practice in this field, is a limitation when tuning models to multiple datasets. It introduces a bias in the $\chi^{2}$ statistic that is difficult to quantify, and requires imposing ad hoc uncertainties [4] as the test statistic is not expected to follow a $\chi^{2}$ distribution for the given degrees of freedom.

The covariance matrices contain a flux-dominated normalization component which we expect to be fully correlated across all distributions. To account for the correlated uncertainty, we use the full covariance matrix, $M_{i j}$, for the $p_{\mu}$ distribution and shape-only covariance matrices, $S_{i j}$, for the other three distributions in each of the topologies. While any distribution could set the normalization constraint, the shape of the $p_{\mu}$ distribution for each channel was chosen since it was found to be relatively insensitive to model variations and had good shape agreement with the data. The joint $\chi^{2}$ is therefore defined as the sum of the full $p_{\mu} \chi^{2}$ and shape-only $\theta_{\mu}, T_{\pi}$, and $\theta_{\pi} \chi^{2}$ 's:

$$
\chi^{2}=\sum_{i j}^{N_{p_{\mu}}} \Delta_{i}\left(M^{-1}\right)_{i j} \Delta_{j}+\sum_{k i j}^{N_{k}} \Delta_{k, i}^{S}\left(S^{-1}\right)_{i j} \Delta_{k, j}^{S},
$$

where $i$ and $j$ are bin indices,

$$
\begin{gathered}
\Delta_{i}=d_{p_{\mu}, i}-m_{p_{\mu}, i} \\
\Delta_{k, i}^{S}=d_{k, i}-\left(m_{k, i} \times \frac{\sum_{j} d_{k, j}}{\sum_{j} m_{k, j}}\right),
\end{gathered}
$$


and $d_{k, i}$ and $m_{k, i}$ are the data and $\mathrm{MC}$ values, respectively, for the $i$ th bin in the $k$ th distribution. The shape-only covariance matrices are provided in the public data release for the $\nu_{\mu} \mathrm{CC} 1 \pi^{ \pm}$and $\nu_{\mu} \mathrm{CC} 1 \pi^{0}$ measurements, and the method of Ref. [21] (Sec. 10.6.3) was used to extract them for the $\nu_{\mu} \mathrm{CCN} \pi^{ \pm}$and $\bar{\nu}_{\mu} \mathrm{CC} 1 \pi^{0}$ channels.
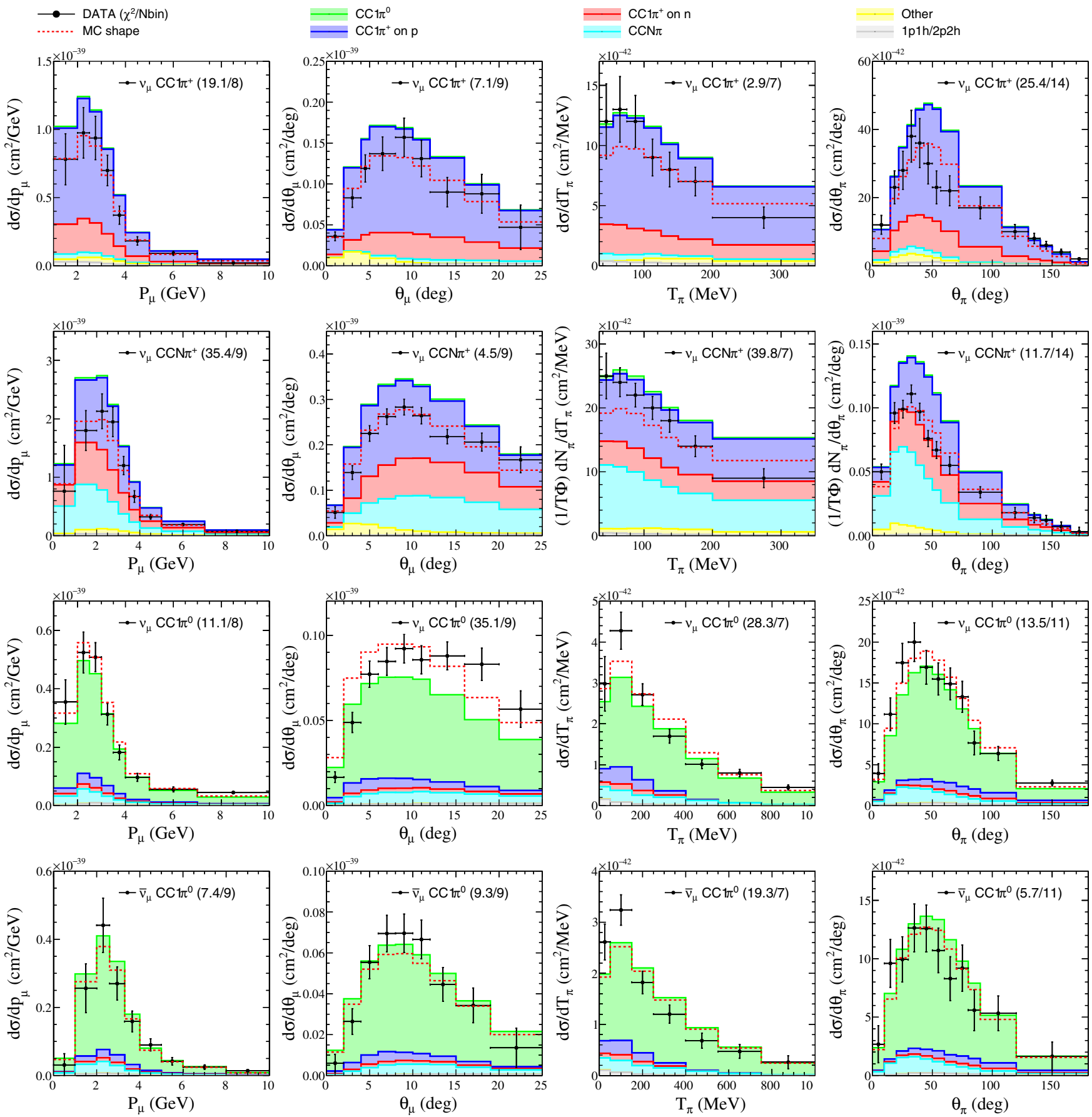

FIG. 1. GENIE 2.12.6 default model predictions compared to MINER $\nu$ A data. Colors correspond to particle content at the nucleon interaction. "Other" is dominated by coherent pion production. "MC shape" shows the total MC prediction after it has been normalized to match the total data normalization. In the case of the shape-only distributions $\left(\theta_{\mu}, T_{\pi}, \theta_{\pi}\right)$ the shape-only $\chi^{2} / N_{\text {bins }}$ values are shown. All cross sections are per nucleon. 
TABLE II. Channel-by-channel contributions to the $\chi^{2}$ at different stages of the tuning process.

\begin{tabular}{lcrrrrr}
\hline \hline \multirow{2}{*}{ Distribution } & Channel & $N_{\text {bins }}$ & Default & BNL & tune & tune \\
\hline$p_{\mu}$ (rate) & $\nu_{\mu} \mathrm{CC} 1 \pi^{ \pm}$ & 8 & 19.1 & 13.8 & 12.0 & 12.3 \\
& $\nu_{\mu} \mathrm{CC} N \pi^{ \pm}$ & 9 & 35.4 & 19.5 & 26.1 & 26.8 \\
& $\nu_{\mu} \mathrm{CC} 1 \pi^{0}$ & 8 & 11.1 & 19.6 & 19.0 & 19.3 \\
& $\bar{\nu}_{\mu} \mathrm{CC} 1 \pi^{0}$ & 9 & 7.4 & 6.4 & 6.2 & 6.3 \\
$\theta_{\mu}$ (shape) & $\nu_{\mu} \mathrm{CC} 1 \pi^{ \pm}$ & 9 & 7.1 & 12.4 & 7.5 & 7.4 \\
& $\nu_{\mu} \mathrm{CC} N \pi^{ \pm}$ & 9 & 4.5 & 10.4 & 4.0 & 4.1 \\
& $\nu_{\mu} \mathrm{CC} 1 \pi^{0}$ & 9 & 35.1 & 71.5 & 44.5 & 45.6 \\
& $\bar{\nu}_{\mu} \mathrm{CC} 1 \pi^{0}$ & 9 & 9.3 & 14.0 & 10.2 & 10.3 \\
$T_{\pi}$ (shape) & $\nu_{\mu} \mathrm{CC} 1 \pi^{ \pm}$ & 7 & 2.9 & 2.6 & 2.5 & 2.3 \\
& $\nu_{\mu} \mathrm{CC} N \pi^{ \pm}$ & 7 & 39.8 & 34.7 & 31.2 & 29.4 \\
& $\nu_{\mu} \mathrm{CC} 1 \pi^{0}$ & 7 & 28.3 & 31.4 & 30.9 & 29.9 \\
& $\bar{\nu}_{\mu} \mathrm{CC} 1 \pi^{0}$ & 7 & 19.3 & 17.9 & 16.6 & 16.0 \\
$\theta_{\pi}$ (shape) & $\nu_{\mu} \mathrm{CC} 1 \pi^{ \pm}$ & 14 & 25.4 & 26.5 & 13.0 & 12.6 \\
& $\nu_{\mu} \mathrm{CC} N \pi^{ \pm}$ & 14 & 11.7 & 11.1 & 6.9 & 6.2 \\
& $\nu_{\mu} \mathrm{CC} 1 \pi^{0}$ & 11 & 13.5 & 15.0 & 8.3 & 8.9 \\
& $\bar{\nu}_{\mu} \mathrm{CC} 1 \pi^{0}$ & 11 & 5.7 & 5.9 & 3.4 & 3.5 \\
Total $\chi^{2}$ & & 148 & 275.6 & 312.7 & 242.3 & 240.7 \\
\hline \hline
\end{tabular}

approximation screening [24] is applied as a weight to quasielastic events. The two-particle two-hole process is simulated using the Valencia model $[11,25]$. MINER $\nu \mathrm{A}$ currently uses a modification of v2.8.4 [19,26,27] with an increased rate for the Valencia two-particle two-hole process; that modification is not used here. An important difference in single pion production between v2.8.x and v2.12. $x$ is the angular distributions of single pion events in the Rein-Sehgal model, discussed below. A sample of $2.5 \times 10^{6}$ events were generated using the MINER $\nu$ A flux predictions [28], a polystyrene target, and the official GENIE 2.12.6 splines [29].

To simulate pion production, GENIE uses the Rein-Sehgal (RS) model [30] with a hadronic invariant mass cut of $W \leq 1.7 \mathrm{GeV}$. Of the 18 resonances in the RS model, the $\Delta(1600)$ and $N(1990)$ were not included due to their unclear experimental status at the time of implementation. Resonance-resonance and resonance-nonresonance interference terms are not included. Lepton mass terms are only included in calculating phase space limits and are neglected when calculating the cross sections. A discussion of the limitations of this simplification can be found in Ref. [31]. In earlier versions-including v2.8.4-the pion-nucleon distribution was isotropic in the resonance rest frame, but was changed in 2.12.x. Here we use the nonisotropic model as our default and reweight to the isotropic distribution, explained later. The RS nonresonant background is not used by GENIE; rather, a deep inelastic scattering (DIS) model is extended to cover that invariant mass region. The DIS model uses the Bodek-Yang parametrization [32], and the Andreopoulos-Gallagher-Kehayias-Yang (AGKY) model to describe hadronization [33]. In the AGKY model, the Koba-Nielsen-Olesen model [34] is used for $W \leq$ $2.3 \mathrm{GeV}$ and PYTHIA [35] is used for $W \geq 3.0 \mathrm{GeV}$, with a smooth transition in between the two, implemented by randomly selecting the results of one model or the other for each event.

In addition to pion production on a single nucleon, it is also possible for a neutrino to produce a pion by scattering coherently off the nucleus. GENIE uses the Rein-Sehgal coherent pion production model [36,37], including the effect of lepton masses in the cross-section calculation. MINER $\nu \mathrm{A}$ has found that the RS coherent pion production model needs to be suppressed by $\sim 50 \%$ at $T_{\pi}<500 \mathrm{MeV}$ to agree with the data [27]. This correction also moves the shape of the $T_{\pi}$ spectrum closer to the predictions of the Berger-Sehgal coherent model [38]. The $\nu_{\mu} \mathrm{CC} 1 \pi^{ \pm}$channel has a small contribution from coherent production in the lowest $Q^{2}$ bins, but the inclusion of this suppression has only a small effect on the MC predictions. To maintain a model similar to that currently being used by MINER $\nu \mathrm{A}$, this suppression is included in the analysis presented from Sec. IV onwards.

The "hA Intranuke" effective cascade model [39] is used to model pion and nucleon FSIs. In this model, the effect of intranuclear scattering is parametrized as a single cascade step applied to each particle emanating from the primary interaction. This model steps hadrons through a nucleus of radius $r \sim A^{1 / 3}$ and a nuclear density function derived from electron scattering data. The hadron's mean free path is determined from tabulated hadron-proton and hadronneutron cross sections [40]. The probability to interact with the nucleus is high; it is, e.g., $\sim 73 \%$ for a pion from an $E_{\nu}=3 \mathrm{GeV}$ quasielastic event in carbon. When a FSI occurs, the possible interactions (absorption, pion production, knockout, charge exchange, elastic scatter) are chosen according to their proportions for iron.

Default GENIE predictions separated by nucleon level interaction channels for the MINER $\nu \mathrm{A}$ data are shown in Fig. 1. The shape of the $p_{\mu}$ distributions agree well with the data for all four measurements. However, the model overestimates the cross section for $\pi^{ \pm}$production, and as a result the $\chi^{2}$ for the $\nu_{\mu} \mathrm{CC} 1 \pi^{ \pm}$and $\nu_{\mu} \mathrm{CCN} \pi^{ \pm}$, given in the fourth column ("Default") of Table II, are large. The model overestimates $\theta_{\mu}$ below $<5^{\circ}$ in the $\pi^{0}$ channels, although it does correctly predict the shape of the $\theta_{\mu}$ distribution in the $\pi^{ \pm}$channels. The model underestimates the production rate at large $\theta_{\mu}$ in $\nu_{\mu} \mathrm{CC} 1 \pi^{0}$. The shape of the $T_{\pi}$ distribution is in larger disagreement for $\nu_{\mu} \mathrm{CCN} \pi^{ \pm}$data than for $\nu_{\mu} \mathrm{CC} 1 \pi^{ \pm}$. Since the $\nu_{\mu} \mathrm{CC} N \pi^{ \pm}$distributions are summed over all identified $\pi^{ \pm}$, redistributing kinetic energy between $\pi^{ \pm}$in events with more than one $\pi^{ \pm}$could resolve some of this tension. The $\pi^{0}$ channels are underpredicted at low $T_{\pi}$. Finally, GENIE predictions are too high in magnitude at $\theta_{\pi} \approx$ $50^{\circ}$ in both the $\nu_{\mu} \mathrm{CC} 1 \pi^{ \pm}$and $\nu_{\mu} \mathrm{CCN} \pi^{ \pm}$channels, and the 

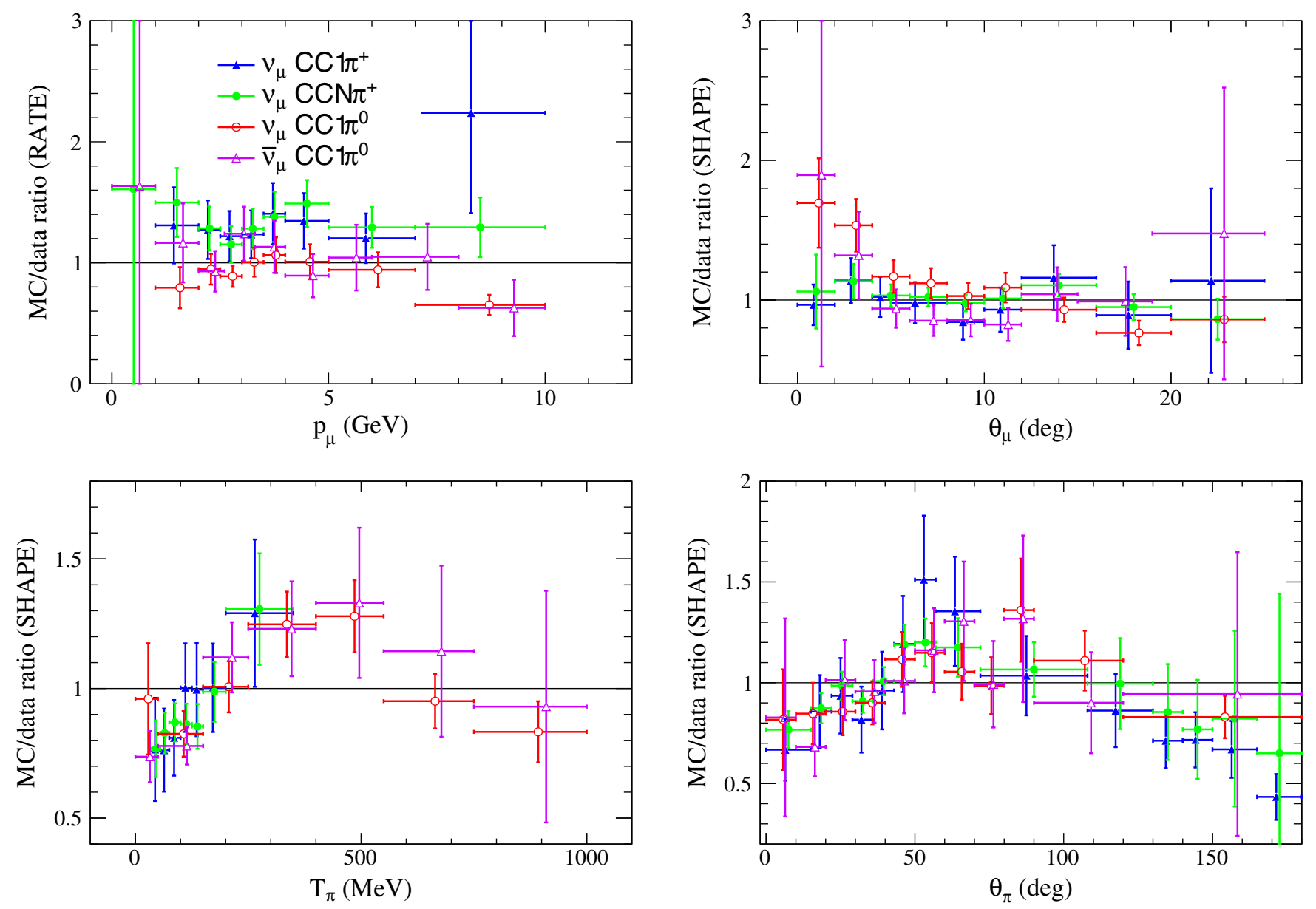

FIG. 2. MC/data ratios for the default GENIE predictions. The $p_{\mu}$ distribution provides a rate comparison in the $\chi^{2}$ calculation; the other distributions are treated as shape only, i.e., the MC is normalized to match the data and the uncertainties are from the shape-only covariance matrix.

prediction has the wrong shape in the $\nu_{\mu} \mathrm{CC} 1 \pi^{ \pm}$channel. Comparisons using the transport theory based Giessen Boltzmann-Uehling-Uhlenbeck (GiBUU) model [41] show similar shape disagreements despite GiBUU's use of an advanced semiclassical cascade model to simulate FSIs [1].

Each of the measurements are shown as MC/data ratio distributions in Fig. 2. Similar comparisons between the MiniBooNE and MINER $\nu \mathrm{A}$ experiments are found in Ref. [5]. The shape-only datasets $\left(\theta_{\mu}, \theta_{\pi}, T_{\pi}\right)$ were normalized to match the data before the ratio was taken and the error bars in Fig. 2 reflect the extracted shape-only uncertainties on the data, so that the distributions reflect their contributions to the total $\chi^{2}$.

\section{TUNABLE PARAMETERS IN THE GENIE MODEL}

The GENIE event generator allows assessment of systematic uncertainties through the GENIE reweighting package. A large number of event weighting "dials" are included to allow model uncertainties to be evaluated. The dials adjusted in this paper are summarized in Table III and are chosen because of their connection to the kinematic variables and interaction modes studied herein.

Experiments often use variations in the charged current resonant axial mass, $M_{\mathrm{A}}^{\text {res }}$, as a systematic uncertainty which varies both the normalization and $Q^{2}$ shape of resonant interactions along with variations in a total resonant cross-section normalization dial, NormRes. Variations in NormRes approximate the behavior of varying $F_{\mathrm{A}}(0)$ in the axial form factor in the Rein-Sehgal model. Since low $\theta_{\mu}$ correlates with low $Q^{2}$, variations in $M_{\mathrm{A}}^{\text {res }}$ have the largest effect on the shape of the muon angular distributions as shown in Fig. 3, and have a small effect on the $\theta_{\pi}$ spectrum.

Dials are available to vary the normalization of the nonresonant $1 \pi$ production channels in GENIE (e.g., NonRESBGvnCC1pi, NonRESBGvpCC1pi) but each dial introduces similar modifications to the predictions. To reduce the number of free parameters in the fit described in Sec. V, these dials were grouped into a single background scaling for nonresonant $1 \pi$ production, NonRes $1 \pi$, following the approach in Refs. $[13,14]$. 
TABLE III. Summary of the GENIE dials optimized in this paper, their default values, and the uncertainties recommended by the GENIE Collaboration. We do not use the defaults for $M_{\mathrm{A}}^{\text {res }}$, NormRes, and NonRes $1 \pi$ and instead impose central values and uncertainties from tunings to ANL and BNL data as described in the text.

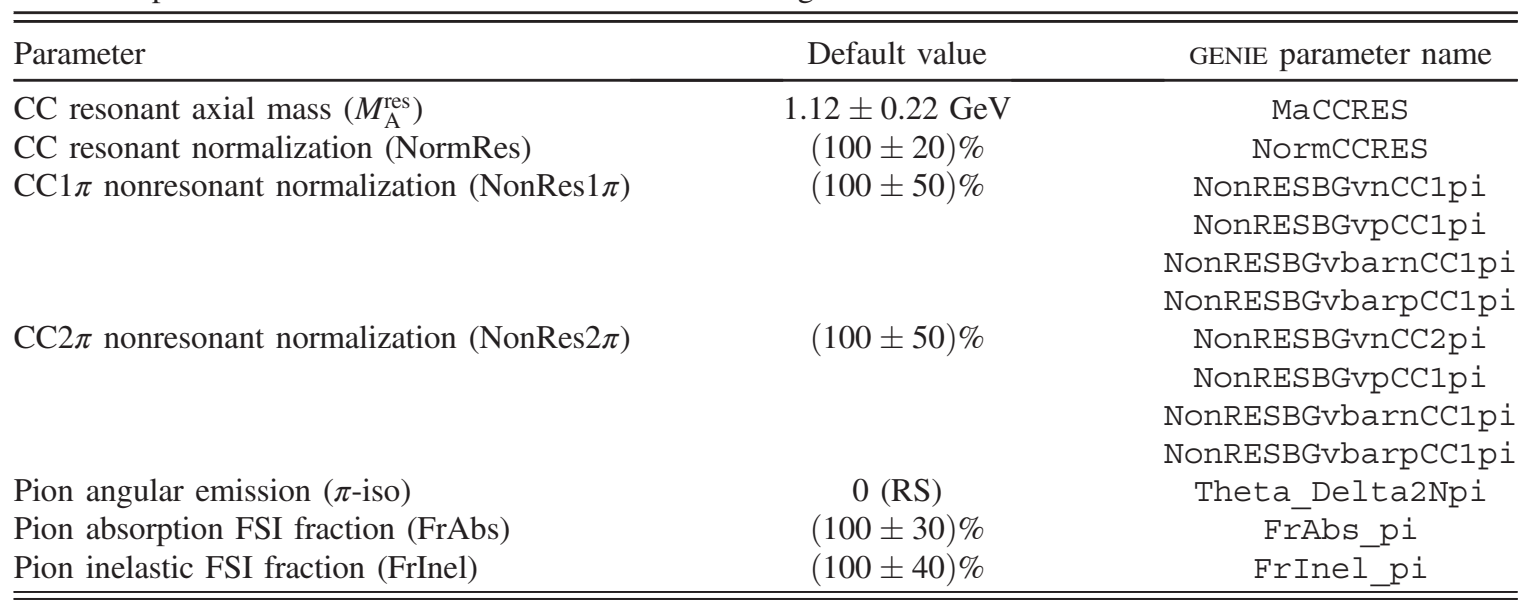

A similar treatment was also applied to nonresonant $2 \pi$ production, NonRes $2 \pi$, with the neutrino and antineutrino related parameters assumed to be $100 \%$ correlated in both cases. The effects of varying the nonresonant contributions are shown in Fig. 4. Variations in the NonRes $2 \pi$ dial introduce a large change in normalization for the $\nu_{\mu} \mathrm{CCN} \pi^{ \pm}$ channel and has a minor effect in the other single pion channels as the fraction of multi- $\pi$ events is small.

Reanalysis of data from ANL and BNL bubble chambers has provided a tuning of GENIE's single pion production model on free nucleons. The work showed that a small shift in $M_{\mathrm{A}}^{\text {res }}$ was required to model the low- $Q^{2}$ region and a large suppression of the nonresonant $\pi$ production $(-54 \%)$ was required to match the observed cross sections of $\pi^{+}$and $\pi^{0}$ production. The reanalysis used the measured ratios of the

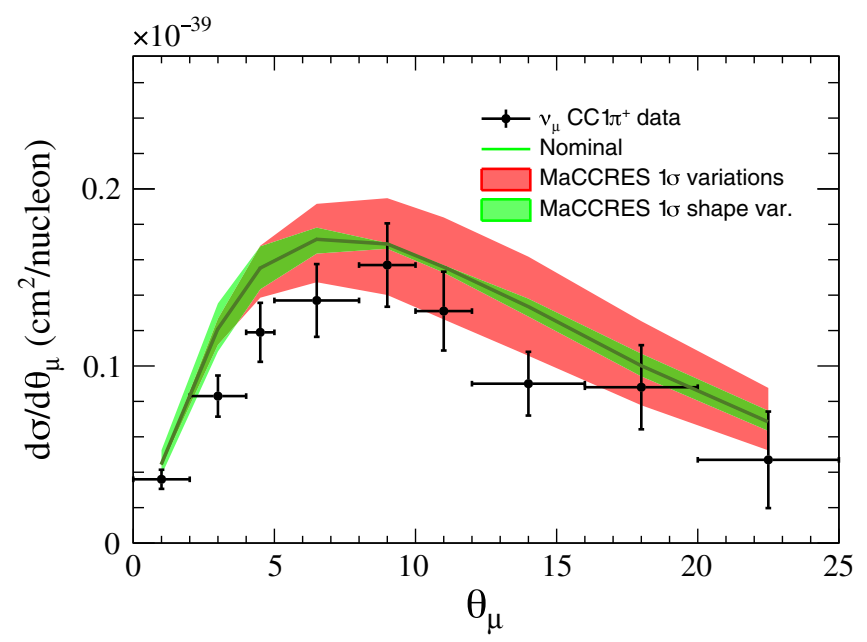

FIG. 3. The effect varying the $M_{\mathrm{A}}^{\text {res }}$ dial on the default GENIE prediction for $\theta_{\mu}$. The red bands show the variation to the total rate and shape. The green bands are obtained by normalizing the reweighted curves to the default predicted rate to highlight the smaller effect the dial has on the shape of the distributions. rates of single $\pi$ production to charged current quasielastic (CCQE) measurements to cancel errors in the flux. We note that by using CCQE data multiple times, they introduce
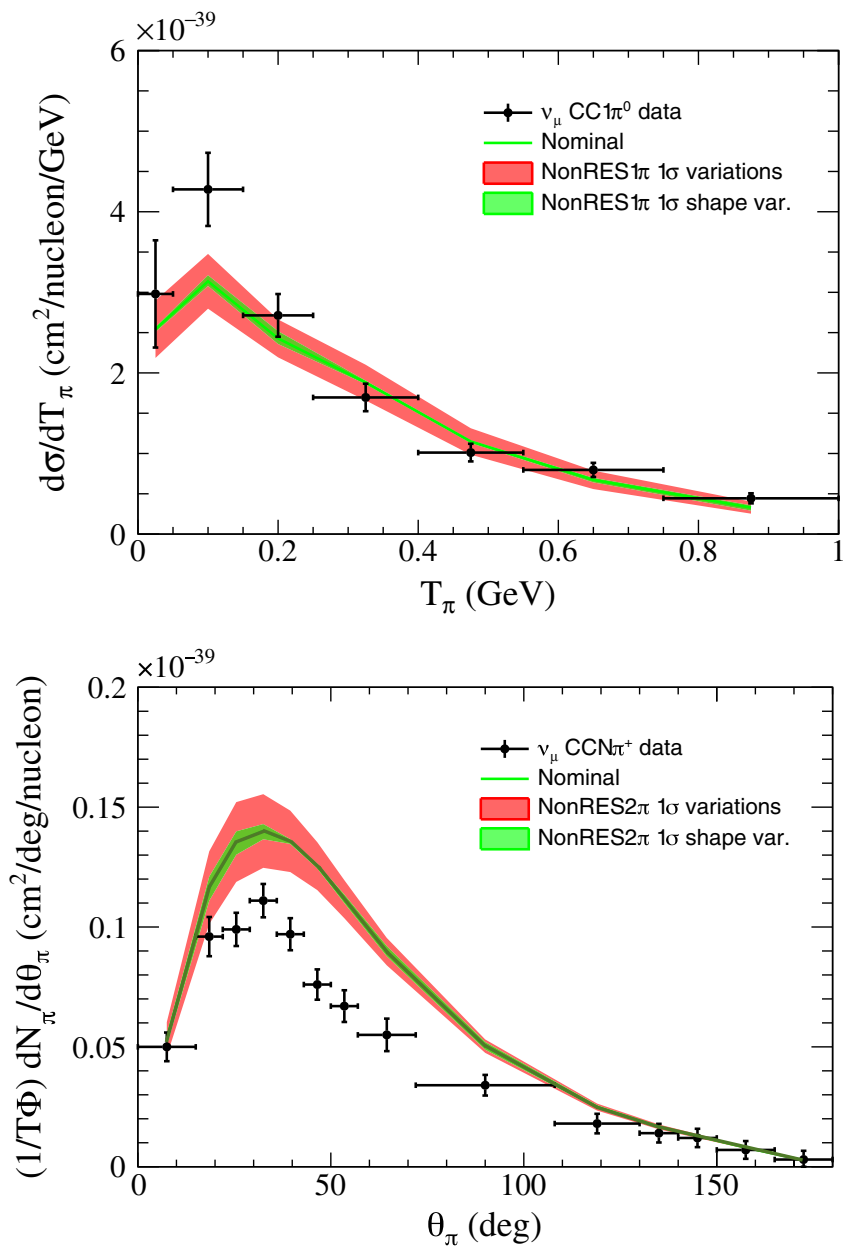

FIG. 4. The effect of varying the NonRes $1 \pi$ dial on the default GENIE prediction for $T_{\pi}$ (top panel) and of varying the NonRes $2 \pi$ dial on the prediction for $\theta_{\pi}$ (bottom panel). 
TABLE IV. Prefit and best-fit central values and uncertainties from tuning GENIE to the ANL/BNL pion production measurements. The prefit uncertainties are those recommended by the GENIE Collaboration. The tuned values are used as penalty terms with the supplied covariance matrix of Fig. 5.

\begin{tabular}{lcc}
\hline \hline Parameter & GENIE default & ANL/BNL tune \\
\hline$M_{\mathrm{A}}^{\text {res }}(\mathrm{GeV})$ & $1.12 \pm 0.22$ & $0.94 \pm 0.05$ \\
NormRes $(\%)$ & $100 \pm 20$ & $115 \pm 7$ \\
NonRes $1 \pi(\%)$ & $100 \pm 50$ & $43 \pm 4$ \\
\hline \hline
\end{tabular}

hidden correlations which may have a small effect on the postfit uncertainties. However, as the single pion statistical uncertainties at ANL [42] and BNL [43] were magnitudes higher than the CCQE statistical uncertainty [44,45], the effect was neglected in that work, and is also neglected here. The resulting parameter tunes shown in Table IV and Fig. 5 have been partially adopted by MINER $\nu \mathrm{A}$ and $\mathrm{NO} \nu \mathrm{A}$ which both apply the nonresonant rescaling of $43 \%$ but leave the other parameters unchanged.

Figure 6 shows MINER $\nu$ A data and the predictions of GENIE when its output has been reweighted to reflect the parameter changes of Table IV. The channel-by-channel contributions to the $\chi^{2}$ are given in the fifth column ("ANL/BNL") of Table II. Incorporating the parameter changes improves the total normalization agreement in the $p_{\mu}$ distributions for $\nu_{\mu} \mathrm{CC} 1 \pi^{ \pm}$and $\nu_{\mu} \mathrm{CCN} \pi^{ \pm}$. The $\chi^{2}$ for the $p_{\mu}$ distribution is also improved in the $\bar{\nu}_{\mu} \mathrm{CC} 1 \pi^{0}$ channel, even though the ANL/BNL data is from neutrino interactions only. The $\chi^{2}$ for the $p_{\mu}$ distribution in the $\nu_{\mu} \mathrm{CC} 1 \pi^{0}$ channel is somewhat worse as the parameter tunes reduce the predicted nucleon $\nu_{\mu} \mathrm{CC} 1 \pi^{0}$ cross section. The modification of $M_{\mathrm{A}}^{\mathrm{res}}$ shifts the $\theta_{\mu}$ predictions to lower values, increasing the $\chi^{2}$ contributions. The $T_{\pi}$ and $\theta_{\pi}$ distributions change mostly by normalization, having a smaller effect on the $\chi^{2}$ ". The overall agreement of GENIE with MINER $\nu \mathrm{A}$ data is not improved by incorporating the ANL/BNL information. Indeed, the total $\chi^{2}$ increases, largely because of the $\chi^{2}$ contributions from the $\theta_{\mu}$ distributions.

GENIE provides a dial that influences the resonances' decay into the pion-nucleon system in the resonance rest frame, $\pi$-iso, and allows events to be reweighted continuously between the default anisotropic distribution $(\pi$-iso $=0)$ and the isotropic distribution $(\pi$-iso $=1)$. The Adler angle ${ }^{2}$ is highly sensitive to the $\pi$-iso parameter and has been measured by neutrino induced pion production experiments on single nucleons, such as ANL [42], BNL [43], BEBC [47,48], and FNAL [49]. Nucleon data strongly prefer an anisotropic process, as shown in Fig. 7. Nonetheless, $\pi$-iso has some impact, albeit one that does depend on how FSI are modeled, on the shape of

\footnotetext{
${ }^{2}$ The Adler angle is the angle between the pion and the threemomentum transfer in the resonance rest frame [46].
}

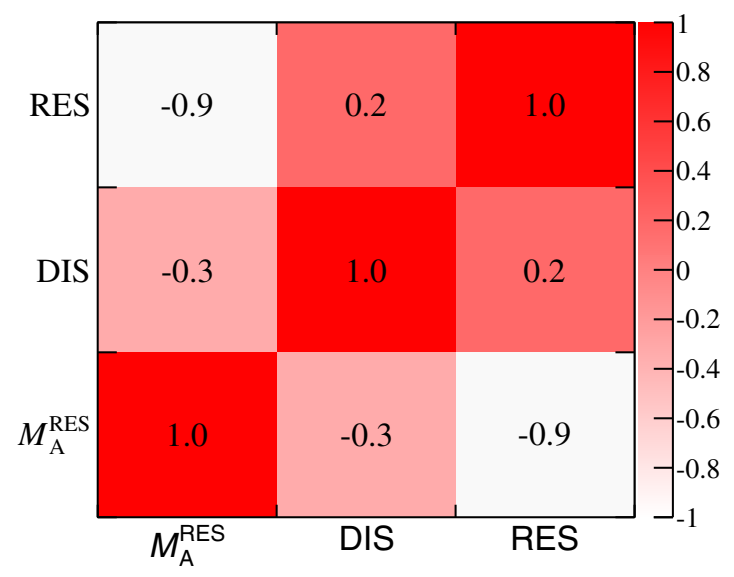

FIG. 5. Correlation matrix from tuning GENIE to reproduce the ANL/BNL pion production measurements included in our $\chi^{2}$ penalty term.

$\operatorname{MINER} \nu \mathrm{A} \theta_{\pi}$ and $T_{\pi}$ distributions, seen at the bottom of Fig. 7, and was therefore included in this work.

The GENIE hA model for FSIs has uncertainties from the $\pi-A$ cross-section data to which the model was tuned. The total $\pi-A$ cross section has a stronger constraint than each of the individual interaction cross sections, so GENIE provides dials to vary the fractional contribution of each component. The available fractional dials are pion absorption (FrAbs), pion inelastic scattering (FrInel), pion elastic scattering, pion charge exchange, and pion production.

\section{TUNING THE GENIE MODEL}

Figure 6 and Table II show the unsatisfactory agreement of the GENIE prediction against MINER $\nu \mathrm{A}$ data. The disagreement worsens after incorporating the prior constraint from ANL and BNL data; this correction, based on nucleon data, is inadequate. This section describes fits that improve the agreement with MINER $\nu \mathrm{A}$ data. The parameters $M_{\mathrm{A}}^{\text {res }}$, NormRes, and NonRes $1 \pi$ are included in the fits with a penalty term added to the $\chi^{2}$ from the ANL and BNL data. The penalty term uses the covariance, $M$, shown in Fig. 5:

$$
\chi_{\text {pen }}^{2}=\sum_{i, j}^{N=3}\left(x_{i}-f_{i}\right)\left(M^{-1}\right)_{i j}\left(x_{j}-f_{j}\right),
$$

where $x_{i}$ are the parameter values $i$ at each iteration of the fit, and $f_{i}$ are the parameter values from the fit to ANL and BNL data. The GENIE default model is strongly disfavored with $\chi_{\text {pen }}^{2}=299.3$, but changing NonRes $1 \pi$ to $43 \%$ while leaving all the other parameters at their default values reduces the $\chi_{\text {pen }}^{2}$ to 21.8 , showing that the largest tension is due to the NonRes $1 \pi$ parameter.

The $\pi$-iso dial is allowed to vary in the range 0 to 1 in the fit, corresponding to a continuous variation between a RS angular distribution and an isotropic distribution for $\Delta(1232)$ decay. To avoid the normalization of the $\nu_{\mu} \mathrm{CCN} \pi^{ \pm}$ 

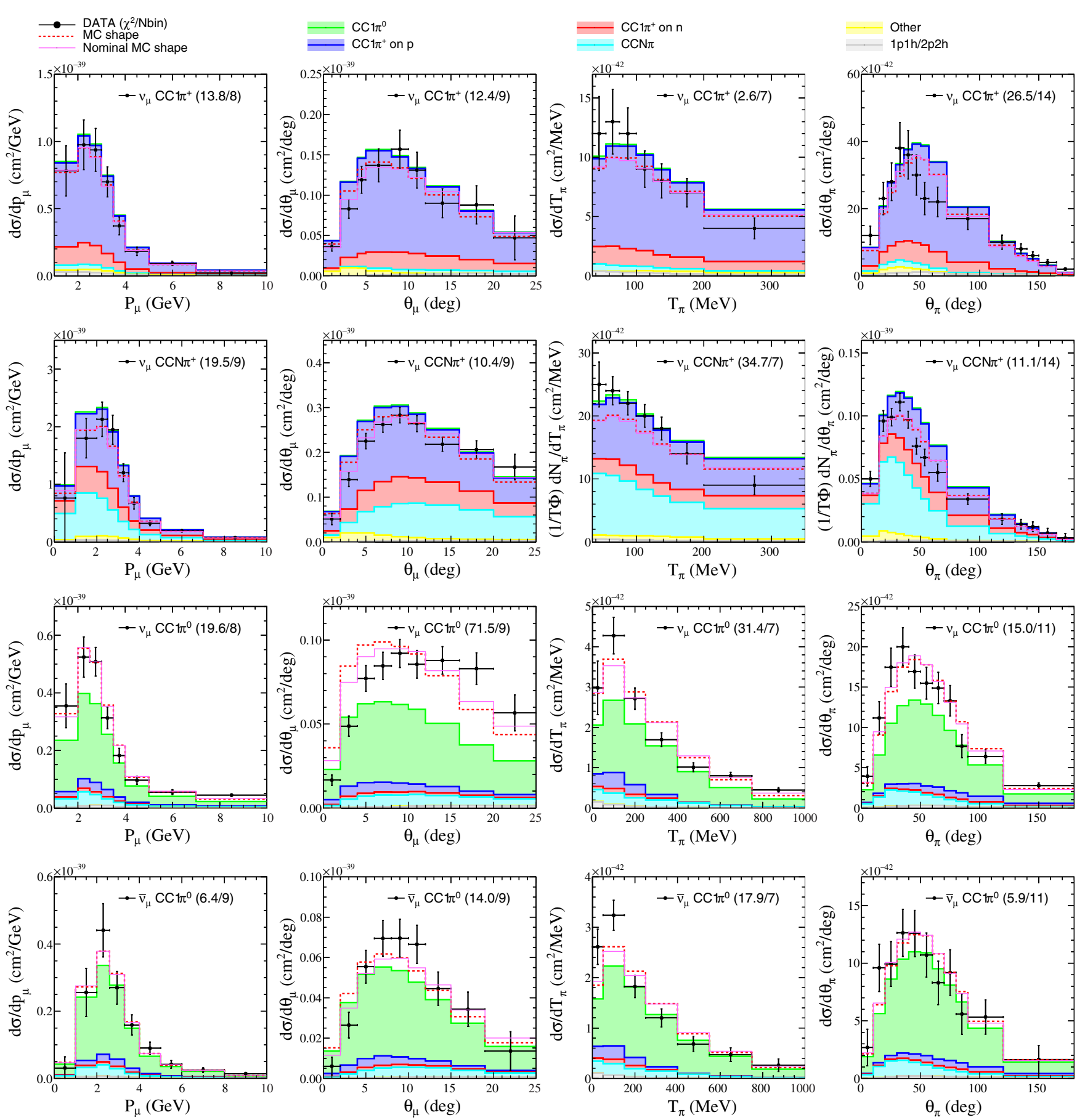

FIG. 6. GENIE ANL/BNL single pion tuning model predictions compared to MINER $\nu$ A data. The distributions have been weighted to the ANL/BNL tuning parameter set, and have had the coherent pion correction applied. Colors correspond to particle content at the nucleon interaction. "Other" is dominated by coherent pion production. "MC shape" shows the total MC prediction after it has been normalized to match the total data normalization. In the case of the shape-only distributions $\left(\theta_{\mu}, T_{\mu}, \theta_{\pi}\right)$ the shape-only $\chi^{2} / N_{\text {bins }}$ values are shown. All cross sections are per nucleon.

measurement pulling parameters in the $\nu_{\mu} \mathrm{CC} 1 \pi^{ \pm}$model, the NonRes $2 \pi$ dial was allowed to vary between $0 \%$ and $300 \%$ of the nominal value.

When varying one of the five hA pion FSI dials, GENIE automatically adjusts the remaining parameters to preserve the total pion cross section and maintain agreement with pion-nucleus scattering data. This "cushion" technique introduced instabilities in the $\chi^{2}$ surface, so it was not possible to include multiple pion FSI parameters in a simultaneous fit. Instead we performed fits with only one of the FSI parameters floating. No $\chi^{2}$ penalty terms were added for the FSI dial in either tuning: the parameters 

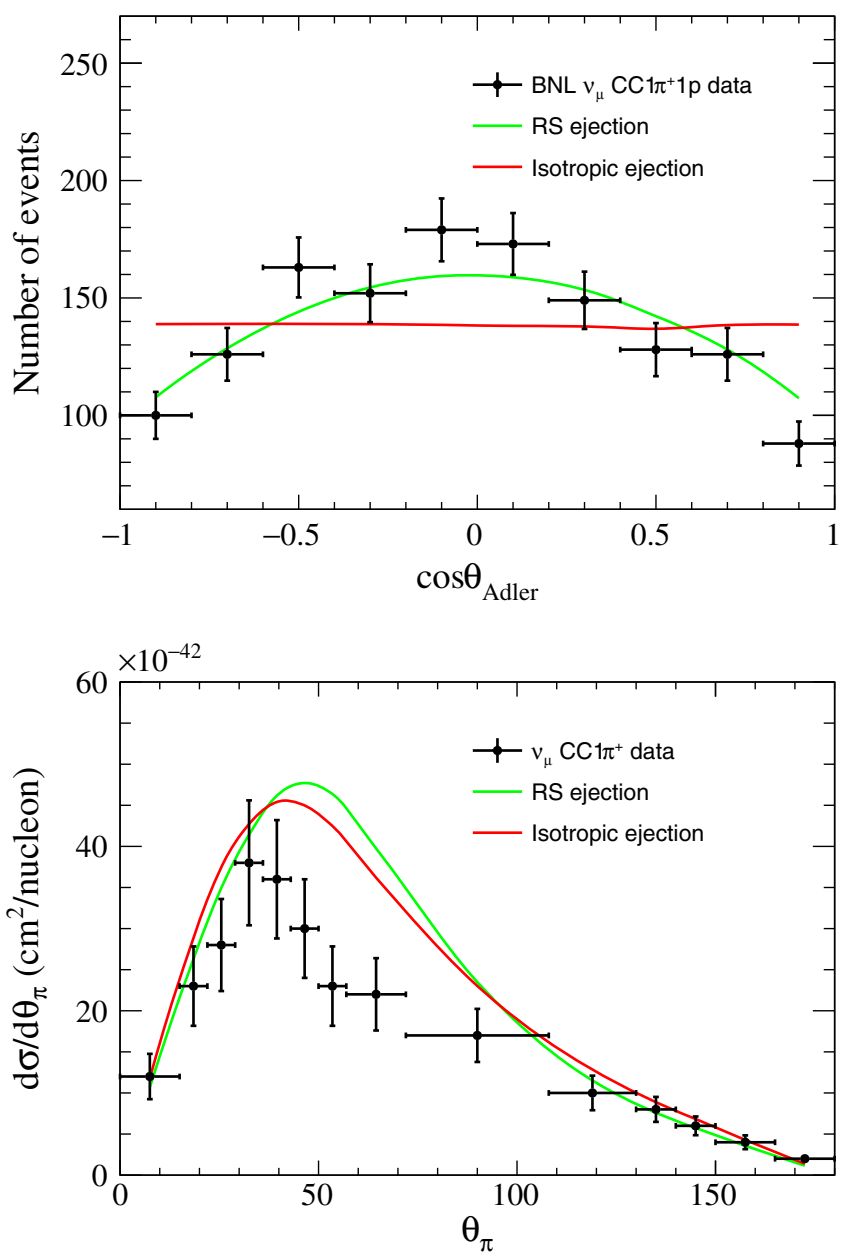

FIG. 7. Effect of varying the $\pi$-iso dial on pion angular distributions for $\mathrm{BNL} \nu_{\mu} \mathrm{CC} 1 \pi^{+} 1 \mathrm{p}$ data (top panel) and $\operatorname{MINER} \nu \mathrm{A} \nu_{\mu} \mathrm{CC} 1 \pi^{+}$(bottom panel).

were driven solely by MINER $\nu \mathrm{A}$ data. The charge exchange and pion production dials had small contributions to the overall $\chi^{2}$ for the selected data, forcing the parameters to be inflated beyond $+3 \sigma$ of GENIE's recommendation, with large postfit uncertainties. Furthermore, the pion elastic scattering parameter is strongly constrained by external data, so its $1 \sigma$ variation has a small impact on the MINER $\nu$ A distributions. The non-FSI fit parameters' (e.g., $M_{\mathrm{A}}^{\text {res }}$ ) central values and uncertainties all agreed for the five fits. Here we present the results from the FrAbs and FrInel fits.

The NUISANCE interface to MINUIT2 [50] was used to perform the fits. At each iteration, the GENIE ReWeight package was used on an event-by-event basis to update the MC predictions before the total $\chi^{2}$ was calculated. The uncertainties in the fitted parameters were determined using the HESSE routine in MINUIT2. The best-fit results from the joint tuning are shown in Table V. Figure 8 shows the ratios of the best-fit prediction to the data for all four kinematic variables of interest when the pion absorption FSI parameter (FrAbs) is floated in the fit; Fig. 9 is the same, but when the inelastic scattering FSI parameter (FrInel) is floated. Notably, the two FSI fits are very similar in both minimum $\chi^{2}$ and best-fit parameter values.

Comparing to the results of the ANL and BNL reanalysis, larger values of $M_{\mathrm{A}}^{\text {res }}$ and smaller values of NormRes were found by the fit, pulling the parameters closer to GENIE nominal. The NonRes $1 \pi$ parameter is strongly bound by the bubble chamber data and the MINER $\nu \mathrm{A}$ data did little to improve on this constraint. The penalty term contributed to the $\chi^{2}$ by 9.3 for the FrAbs fit and 11.1 for the FrInel fit. This is a significant improvement over the default, but indicates that there is mild tension between the nucleon and nuclear data. The postfit correlation matrices are provided in Fig. 10. The ANL/BNL input correlations are largely maintained in our fit.

Tables VI and VII show the results when individual MINER $\nu A$ data were tuned in separate fits. Since three of the four channels were removed in these fits, the constraint from data is weakened and the total $\chi^{2}$ is steered by the bubble chamber $\chi^{2}$ penalty. The individual channel fits also found values at the $300 \%$ limit for NonRes $2 \pi$ dial, except

TABLE V. Fit results from tuning GENIE parameters in NUISANCE. The "ANL/BNL value" column shows the contributions when parameters are fixed at values of Table IV.

\begin{tabular}{lcccc}
\hline \hline Parameter & Default value & ANL/BNL value & FrAbs fit result & FrInel result \\
\hline$M_{\mathrm{A}}^{\text {res }}(\mathrm{GeV})$ & $1.12 \pm 0.22$ & $0.94 \pm 0.05$ & $1.07 \pm 0.04$ & $1.08 \pm 0.04$ \\
NormRes $(\%)$ & $100 \pm 20$ & $115 \pm 7$ & $94 \pm 6$ & $92 \pm 6$ \\
NonRes $1 \pi(\%)$ & $100 \pm 50$ & $43 \pm 4$ & $44 \pm 4$ & $44 \pm 4$ \\
NonRes $2 \pi(\%)$ & $100 \pm 50$ & $\ldots$ & $166 \pm 32$ & $161 \pm 33$ \\
$\pi$-iso & $0=\mathrm{RS}$ & $\ldots$ & $109 \pm 16$ & $1=$ Iso (limit) \\
FrAbs $(\%)$ & $100 \pm 30$ & $\ldots$ & $\ldots$ & $\ldots$ \\
FrInel $(\%)$ & $100 \pm 40$ & $\ldots$ & 242.3 & $109 \pm 24$ \\
MINER $\nu \mathrm{A} \chi^{2}$ & 275.6 & 312.7 & 9.3 & 240.7 \\
$\chi_{\text {pen }}^{2}$ & 299.3 & 0.0 & 251.6 & 11.1 \\
Total $\chi^{2}$ & 574.8 & 312.7 & 145 & 251.8 \\
$N_{\text {DoF }}$ & 148 & 148 & & 145 \\
\hline \hline
\end{tabular}



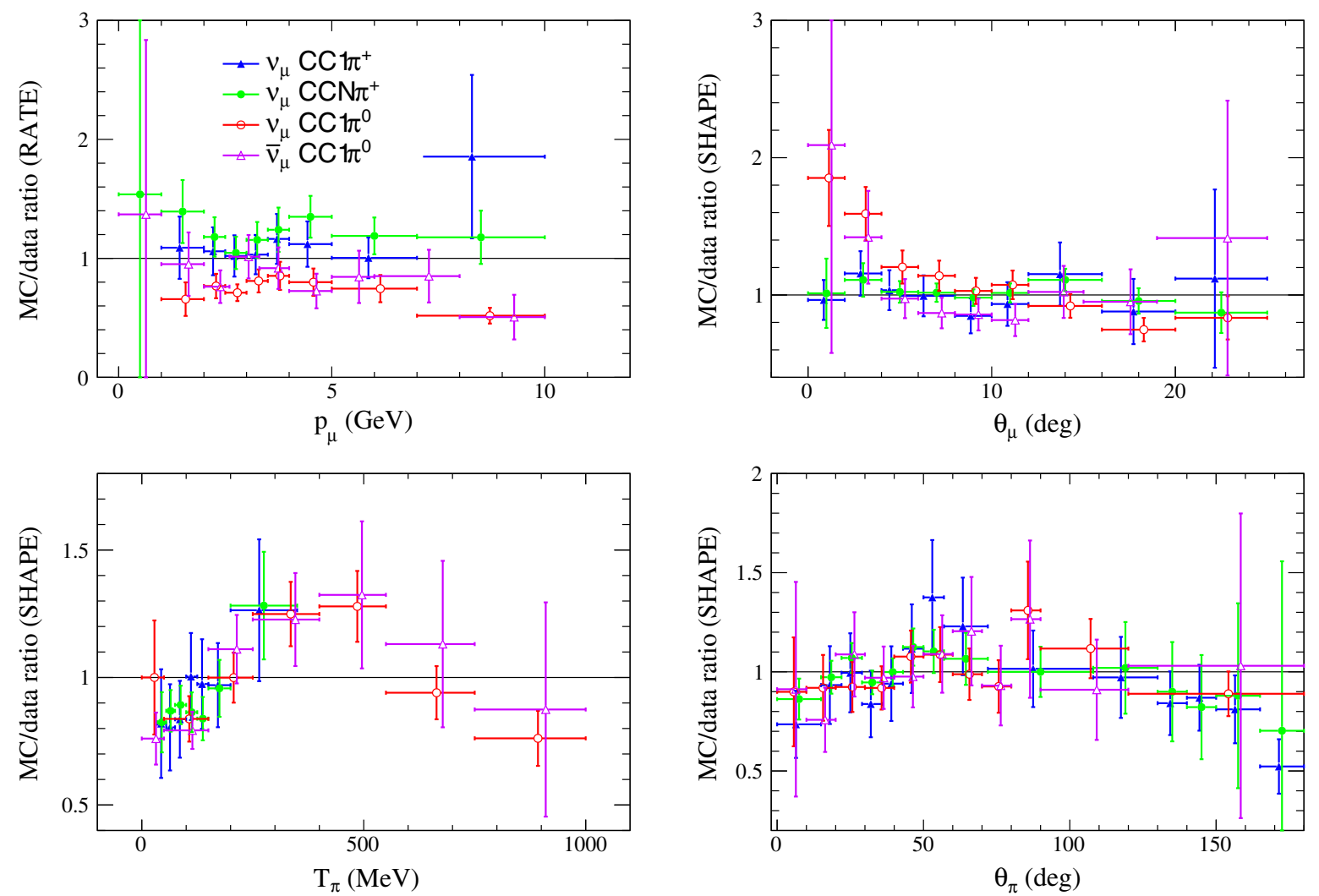

FIG. 8. MC/data ratios at the best-fit point for the FrAbs joint tuning.
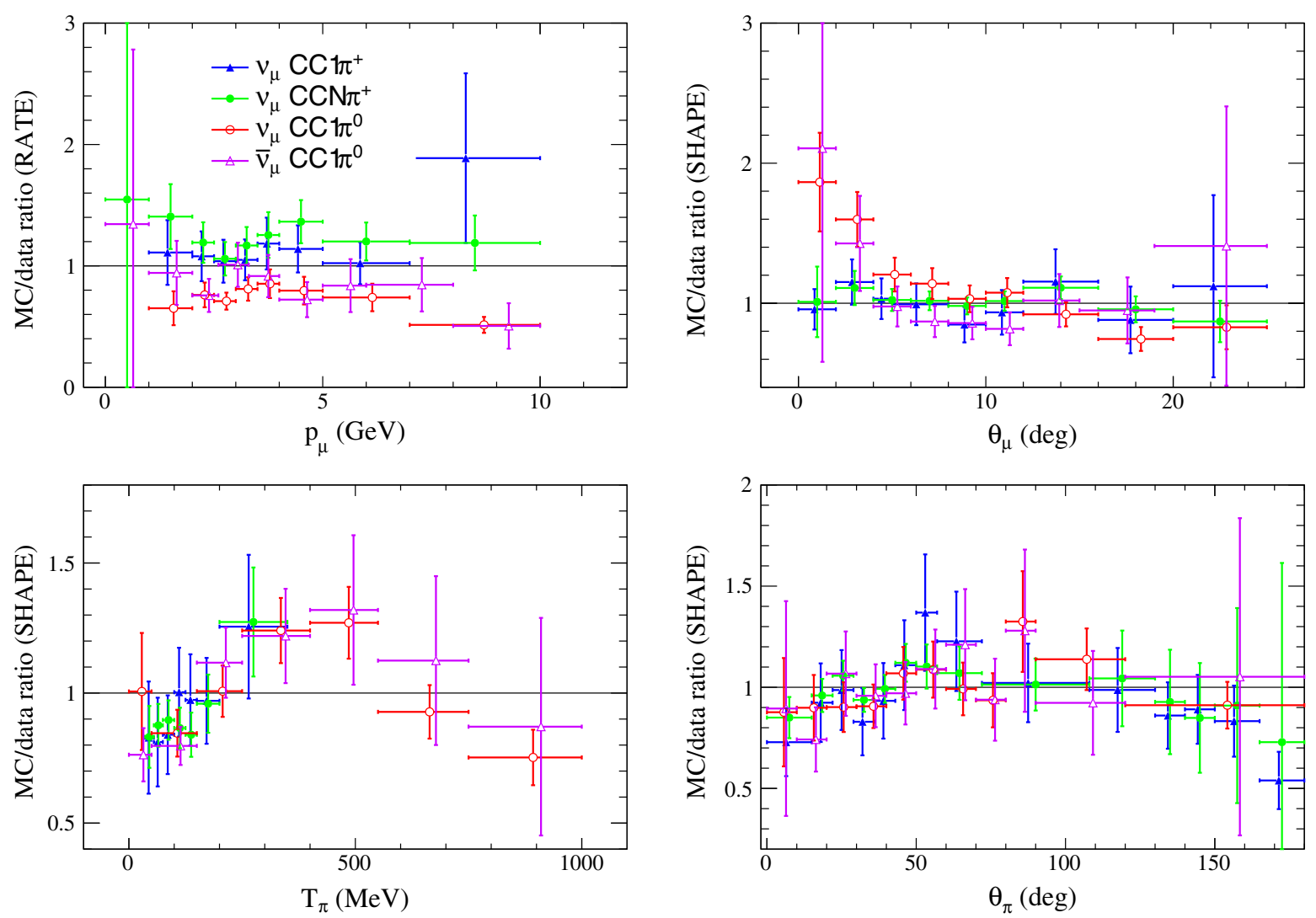

FIG. 9. MC/data ratios at the best-fit point for the FrInel joint tuning. 

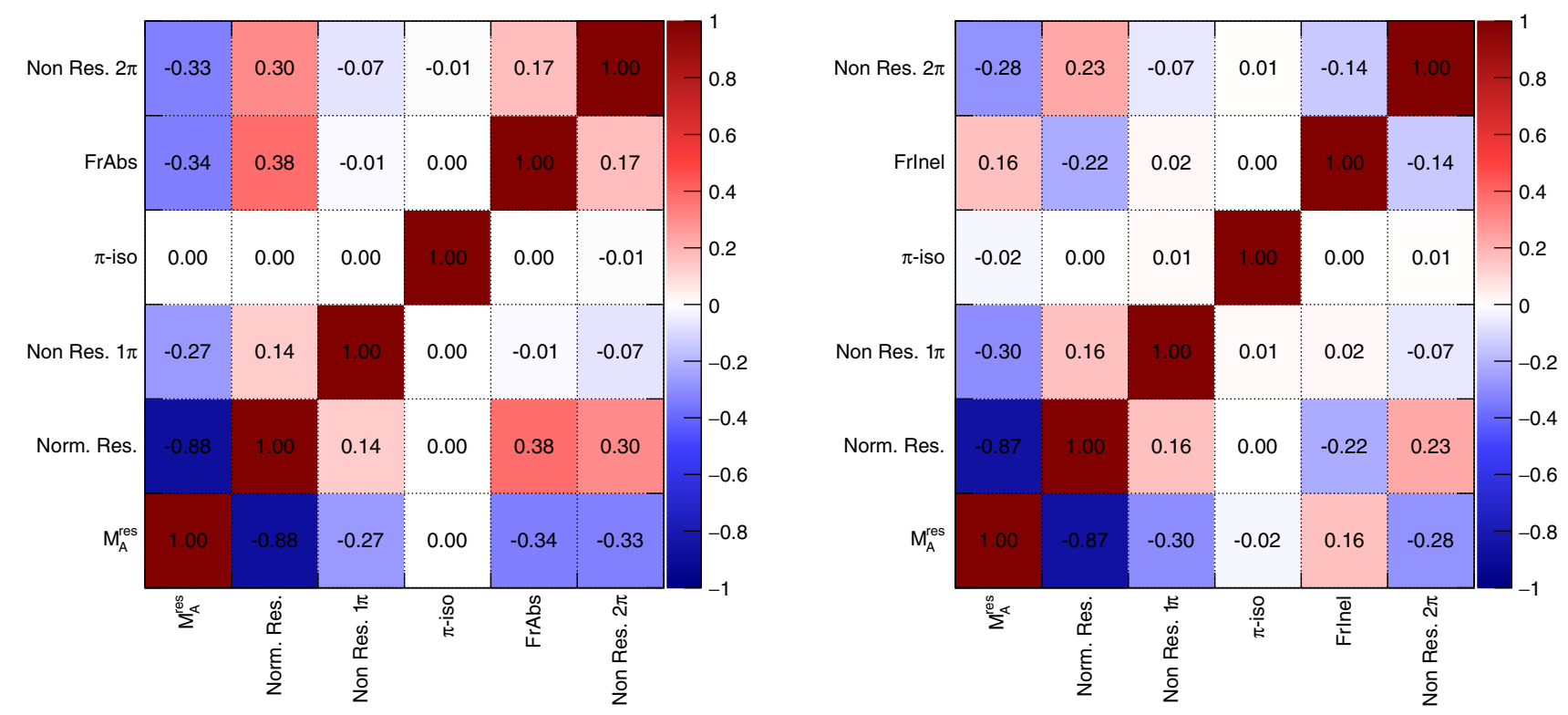

FIG. 10. Correlation matrix from tuning GENIE parameters in NUISANCE with FrAbs included as a fit parameter (left panel) and with FrInel included as a fit parameter (right panel).

in the $\nu_{\mu} \mathrm{CCN} \pi^{ \pm}$channel, where the result was unchanged by the fit. Only the $\nu_{\mu} \mathrm{CCN} \pi^{ \pm}$channel has a significant contribution from nonresonant $2 \pi^{ \pm}$production. In the other fits, the parameter is largely unconstrained and has little impact on the fitted distributions. The $\chi^{2}$ per degree of freedom is indicative of a poor fit in the $\nu_{\mu} \mathrm{CCN} \pi^{ \pm}$and

TABLE VI. Individual channel tuning results when the FrAbs dial is treated as the free FSI parameter.

\begin{tabular}{lcccc}
\hline \hline Parameter & $\nu_{\mu} \mathrm{CC} 1 \pi^{ \pm}$ & $\nu_{\mu} \mathrm{CCN} \pi^{ \pm}$ & $\nu_{\mu} \mathrm{CC} 1 \pi^{0}$ & $\bar{\nu}_{\mu} \mathrm{CC} 1 \pi^{0}$ \\
\hline$M_{\mathrm{A}}^{\text {res }}(\mathrm{GeV})$ & $0.97 \pm 0.05$ & $0.97 \pm 0.05$ & $1.02 \pm 0.05$ & $0.96 \pm 0.05$ \\
NormRes $(\%)$ & $110 \pm 7$ & $110 \pm 7$ & $104 \pm 7$ & $111 \pm 7$ \\
NonRes $1 \pi(\%)$ & $43 \pm 4$ & $42 \pm 4$ & $44 \pm 4$ & $43 \pm 4$ \\
NonRes $2 \pi(\%)$ & 300 (limit) & $99 \pm 30$ & 300 (limit) & 300 (limit) \\
$\pi$-iso & $1=$ Iso (limit) & $1=$ Iso (limit) & $1=$ Iso (limit) & $1=$ Iso (limit) \\
FrAbs $(\%)$ & $156 \pm 53$ & $128 \pm 34$ & $126 \pm 17$ & $82 \pm 31$ \\
MINER $\nu \mathrm{A} \chi^{2}$ & 36.6 & 64.1 & 92.3 & 34.6 \\
$\chi_{\text {pen }}^{2}$ & 0.5 & 0.7 & 3.2 & 0.3 \\
Total $\chi^{2}$ & 37.1 & 64.8 & 95.5 & 34.9 \\
$N_{\text {DoF }}$ & 35 & 36 & 32 & 33 \\
\hline \hline
\end{tabular}

TABLE VII. Individual channel tuning results when the FrInel dial is treated as the free FSI parameter.

\begin{tabular}{lcccc}
\hline \hline Parameter & $\nu_{\mu} \mathrm{CC} 1 \pi^{ \pm}$ & $\nu_{\mu} \mathrm{CCN} \pi^{ \pm}$ & $\nu_{\mu} \mathrm{CC} 1 \pi^{0}$ & $\bar{\nu}_{\mu} \mathrm{CC} 1 \pi^{0}$ \\
\hline$M_{\mathrm{A}}^{\text {res }}(\mathrm{GeV})$ & $0.97 \pm 0.05$ & $0.97 \pm 0.05$ & $1.03 \pm 0.05$ & $0.96 \pm 0.05$ \\
NormRes $(\%)$ & $109 \pm 7$ & $108 \pm 7$ & $103 \pm 7$ & $112 \pm 7$ \\
NonRes1 $\pi(\%)$ & $42 \pm 4$ & $42 \pm 4$ & $43 \pm 4$ & $43 \pm 4$ \\
NonRes $2 \pi(\%)$ & 300 (limit) & $110 \pm 30$ & 300 (limit) & 300 (limit) \\
$\pi$-iso & $1=$ Iso (limit) & $1=$ Iso (limit) & $1=$ Iso (limit) & $1=$ Iso (limit) \\
FrInel $(\%)$ & $117 \pm 54$ & $127 \pm 33$ & 0 (limit) & $80 \pm 59$ \\
MINER $\nu \mathrm{A} \chi^{2}$ & 37.1 & 63.4 & 86.9 & 34.9 \\
$\chi_{\text {pen }}^{2}$ & 0.7 & 1.3 & 3.4 & 0.2 \\
Total $\chi^{2}$ & 37.8 & 64.7 & 90.3 & 35.1 \\
$N_{\text {DoF }}$ & 35 & 36 & 32 & 33 \\
\hline \hline
\end{tabular}


$\nu_{\mu} \mathrm{CC} 1 \pi^{0}$ channels, but not in the $\nu_{\mu} \mathrm{CC} 1 \pi^{ \pm}$or $\bar{\nu}_{\mu} \mathrm{CC} 1 \pi^{0}$ channels. Furthermore, the $\nu_{\mu} \mathrm{CC} 1 \pi^{0}$ shows the strongest $\chi^{2}$ penalty, indicating tension with the ANL/BNL prior. Given the different kinematic regions covered by the channels (see Table I) and the different physics (e.g., fraction of coherent pion production) it is difficult to infer what combination of effects are at work. Isotropic emission was preferred in all fits, driven by the $\theta_{\pi}$ distributions. Disagreements in the $\theta_{\pi}$ spectrum are clearly seen in the data/MC ratios of Figs. 8 and 9, and the large $\chi^{2}$ values observed for the $\nu_{\mu} \mathrm{CCN} \pi^{ \pm}$and $\nu_{\mu} \mathrm{CC} 1 \pi^{0}$ channels.

The individual $\chi^{2}$ contributions in the joint tuning best fit, shown in sixth and seventh columns ("FrAbs tune" and "FrInel tune") of Table II, show that not all distributions in all channels benefit from the model variations, as the default GENIE fits have a better $\chi^{2}$ for some distributions.
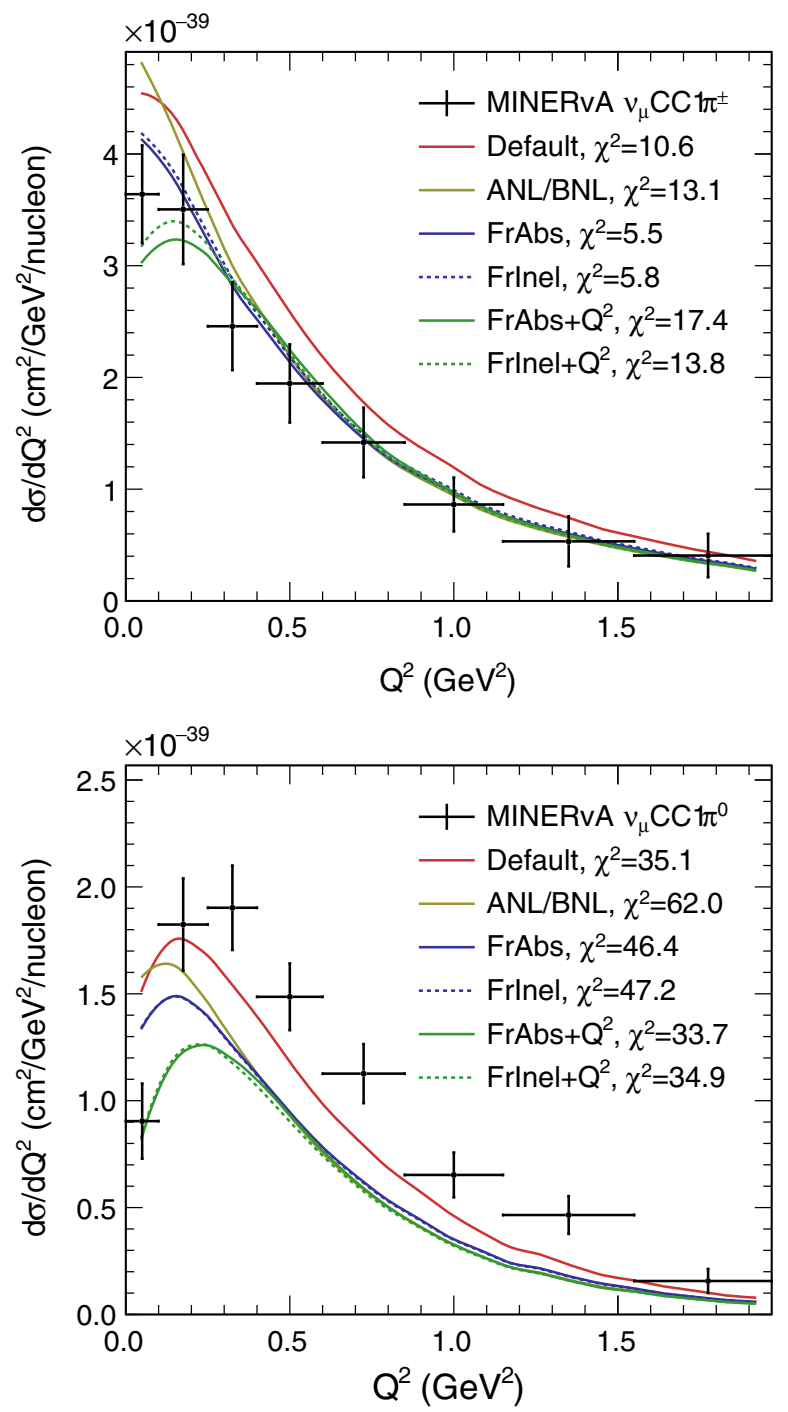

In particular, the $\nu_{\mu} \mathrm{CC} 1 \pi^{0}$ channel distributions have worse agreement after the tuning, with only the $\theta_{\pi}$ distribution improving in $\chi^{2}$, whereas all channels benefit from the shift to isotropic emission. While there is an overall improvement over the ANL/BNL tune when comparing the combined $\chi^{2}$ results, Figs. 8 and 9 show that there are still unresolved shape disagreements in both the $T_{\pi}$ and $\theta_{\mu}$ kinematics.

The tension between MINER $\nu$ A's nuclear data and the constraints from the ANL and BNL nucleon data is difficult to confidently pinpoint; the lack of lepton mass effects [51], modification to the resonance propagator in the nucleus [52,53], missing diagrams describing the nonresonant background contributions [9], dynamical coupled channels [54], interactions on correlated initial states, and the pion FSI model [1] are all part of an incomplete list of possible culprits.
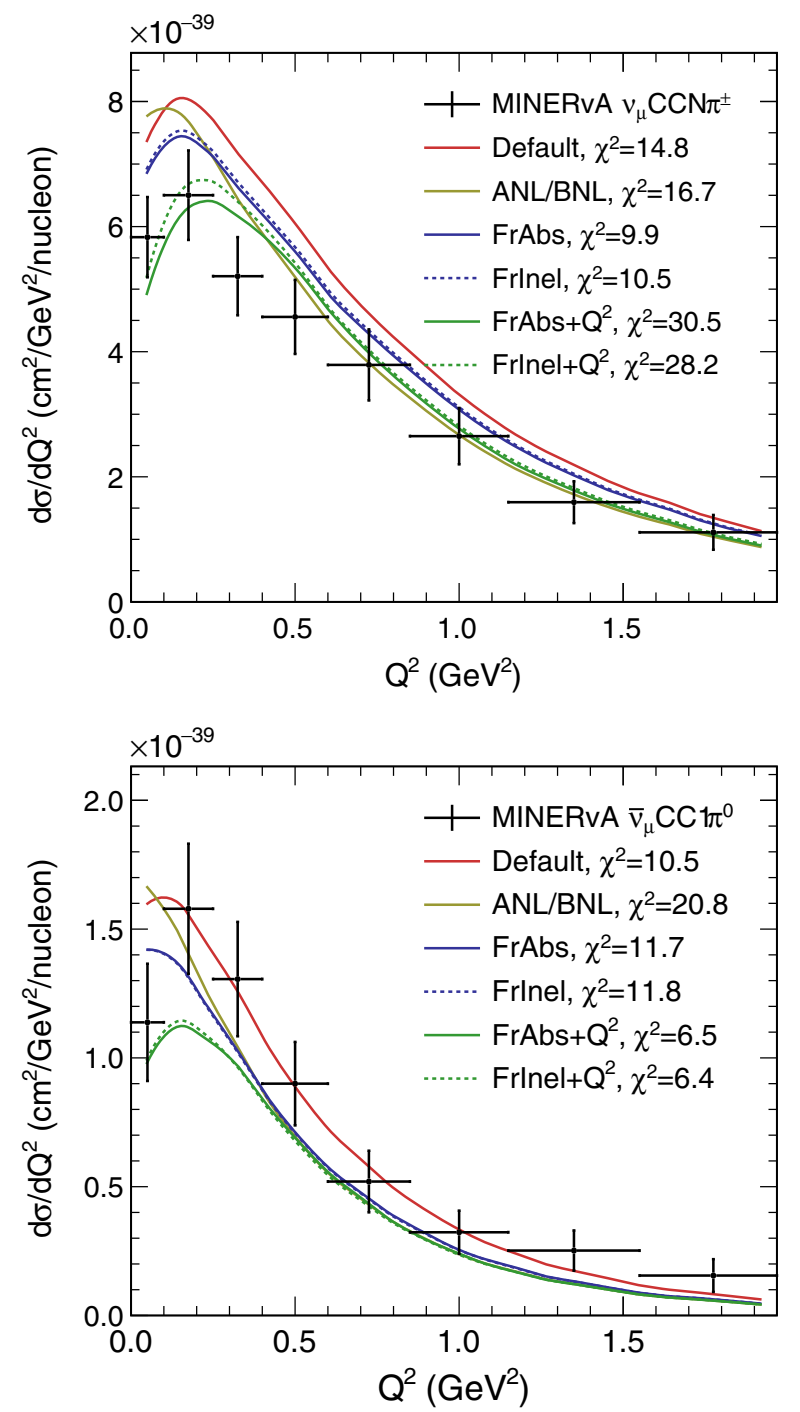

FIG. 11. Comparisons of the nominal and tuned models to MINER $\nu \mathrm{A} \nu_{\mu} \mathrm{CC} 1 \pi^{ \pm}$(left top panel), $\nu_{\mu} \mathrm{CCN} \pi^{ \pm}$(right top panel), $\nu_{\mu} \mathrm{CC} 1 \pi^{0}$ (left bottom panel) and $\bar{\nu}_{\mu} \mathrm{CC} 1 \pi^{0}$ (right bottom panel) distributions in $Q^{2}$. The $\chi^{2}$ is computed using the full covariance matrices. The distributions were not explicitly used in the tuning procedure. 


\section{Ad hoc $Q^{2}$ SUPRESSION}

Further modifications beyond the standard GENIE dials are required to resolve the observed tensions. Figure 11 (not used for any tuning) shows the $Q^{2}$ distributions observed at MINER $\nu \mathrm{A}$ for our tunes. The data are below the predictions of the tunes of Sec. V at low values of $Q^{2}$. There are also differences at low $\theta_{\mu}$, as shown in Figs. 8 and 9. Measurements of $\nu_{\mu} \mathrm{CC} 1 \pi^{ \pm}$and $\nu_{\mu} \mathrm{CC} 1 \pi^{0}$ interactions on mineral oil at MiniBooNE have shown a data/MC shape discrepancy for the RS implementation in the NUANCE model $[55,56]$ in both $Q^{2}$ and $\cos \theta_{\mu}$ distributions [16,57]. In the MINOS quasielastic analysis [15] on iron, which used NeuGen [58], a similar disagreement was observed when studying pion production dominated sidebands. Indeed, concerns about low- $Q^{2}$ modeling date back almost a decade [31]. The data from MINOS and MiniBooNE experiments and the MINER $\nu \mathrm{A}$ data on polystyrene studied herein suggest that the RS implementation common to each of the generators needs to be suppressed at low $Q^{2}$. Collective effects, which are usually modeled in the random phase approximation, are known to affect the $Q^{2}$ distribution of neutrino-nucleus reactions at low $Q^{2}$. Motivated by these considerations, we attempted to improve the $\theta_{\mu}$ modeling by introducing a $Q^{2}$-dependent correction to the model.

The MINOS Collaboration suppression was expressed as

$$
R=\frac{A}{1+\exp \left\{1-\sqrt{Q^{2}} / Q_{0}\right\}},
$$

where the free parameters $A=1.010$ and $Q_{0}=0.156 \mathrm{GeV}$ were empirically extracted from bin-by-bin fits in $Q^{2}$ to the data, and a hard cutoff at $Q^{2}<0.7 \mathrm{GeV}^{2}$ was imposed.

We chose an empirical function so that the shape of the suppression preferred by each of the MINER $\nu \mathrm{A}$ channels could be extracted. The empirical correction function is applied to events with a resonance decay inside the nucleus giving rise to a pion. Our suppression term is defined by choosing three points $\left(x_{i}, R_{i}\right)_{i=1,2,3}$ between $0.0<x<1.0$ and $0.0<R<1.0$, where $x \equiv Q^{2}$. Motivated the ANL/BNL curves in Fig. 11, the correction is assumed to approach unity as $Q^{2}$ approaches $0.7 \mathrm{GeV}^{2}$, providing the constraint $\left(x_{3}, R_{3}\right)=\left(0.7 \mathrm{GeV}^{2}, 1.0\right)$. Lagrange interpolation is used to derive a curvature from $R_{2}$ by assuming a simple interpolation between the points $\left(x_{1}, 0.0\right),\left(x_{2}, R_{2}\right)$, and $\left(0.7 \mathrm{GeV}^{2}, 1.0\right)$ :

$$
\begin{aligned}
R\left(Q^{2}<x_{3}\right)= & \frac{R_{2}\left(Q^{2}-x_{1}\right)\left(Q^{2}-x_{3}\right)}{\left(x_{2}-x_{1}\right)\left(x_{2}-x_{3}\right)} \\
& +\frac{\left(Q^{2}-x_{1}\right)\left(Q^{2}-x_{2}\right)}{\left(x_{3}-x_{1}\right)\left(x_{3}-x_{2}\right)} .
\end{aligned}
$$

This interpolation function is then used to calculate the correction for each event as

$$
w\left(Q^{2}\right)=1-\left(1-R_{1}\right)\left(1-R\left(Q^{2}\right)\right)^{2},
$$

where $R_{1}$ defines the magnitude of the correction function at the intercept, $x_{1}=0.0 . x_{2}$ is chosen to be $Q^{2}=$ $0.35 \mathrm{GeV}^{2}$ so that $R_{2}$ describes the curvature at the center point of the correction. Expressing the weights with Eqs. (6) and (7) ensures that the magnitude at $x_{2}$ always lies between $R_{1}$ and 1.0, avoiding parameter sets with large unphysical peaks in the correction function. Additionally, the squared term in Eq. (7) ensures that $w\left(Q^{2}\right) \rightarrow 1.0$ as $x \rightarrow x_{3}$, avoiding discontinuous steps in the weighting function at $x_{3}$. The fitted parameters $R_{1}$ and $R_{2}$ were limited to $0.0<R_{1}<1.0$ and $0.5<R_{2}<1.0$ to avoid extraneous solutions, e.g., double peaks.

The fit results are shown in Table VIII. The correction from the fit with FrAbs taken as a free parameter are

TABLE VIII. Ad hoc low- $Q^{2}$ suppression model tuning results compared to the tuning results without the low- $Q^{2}$ suppression.

\begin{tabular}{lcccc}
\hline \hline Parameter & FrAbs tune & FrAbs + low- $Q^{2}$ tune & FrInel tune & FrInel + low- $Q^{2}$ tune \\
\hline$M_{\mathrm{A}}^{\text {res }}(\mathrm{GeV})$ & $1.07 \pm 0.04$ & $0.92 \pm 0.02$ & $1.08 \pm 0.04$ & $0.93 \pm 0.05$ \\
NormRes $(\%)$ & $94 \pm 6$ & $116 \pm 3$ & $92 \pm 6$ & $116 \pm 7$ \\
NonRes $1 \pi(\%)$ & $43 \pm 4$ & $46 \pm 4$ & $44 \pm 4$ & $46 \pm 4$ \\
NonRes $2 \pi(\%)$ & $166 \pm 32$ & $99 \pm 31$ & $161 \pm 33$ & $120 \pm 32$ \\
$\pi$-iso & 1.0 (limit) & 1.0 (limit) & 1.0 (limit) & 1.0 (limit) \\
FrAbs $(\%)$ & $109 \pm 16$ & $48 \pm 21$ & $\ldots$ & $\ldots$ \\
FrInel $(\%)$ & $\ldots$ & $\ldots .32 \pm 0.06$ & $109 \pm 24$ & $132 \pm 27$ \\
Lag. $R_{1}$ & $\ldots$ & 0.5 (limit) & $\ldots$ & $0.37 \pm 0.09$ \\
Lag. $R_{2}$ & $\ldots$ & 212.2 & 240.7 & $0.60 \pm 0.16$ \\
MINER $\nu \mathrm{A} \chi^{2}$ & 242.3 & 0.7 & 11.1 & 215.7 \\
$\chi_{\text {pen }}^{2}$ & 9.3 & 212.9 & 251.8 & 0.5 \\
Total $\chi^{2}$ & 251.6 & 143 & 145 & 216.2 \\
$N_{\text {DoF }}$ & 145 & & & 143 \\
\hline \hline
\end{tabular}



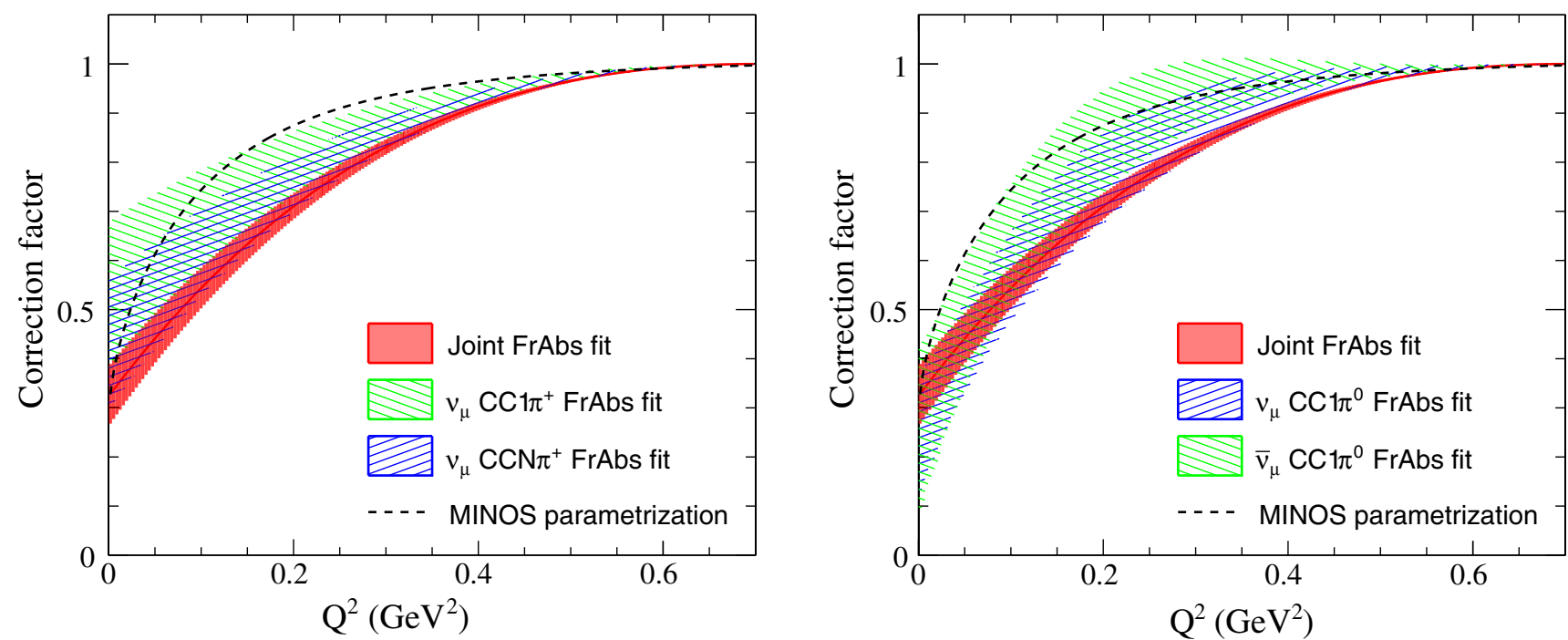

FIG. 12. Extracted low- $Q^{2}$ suppression factors from the FrAbs + low- $Q^{2}$ tuning to each channel. The left and right plots compare the results for the charged and neutral pion production channels, respectively. Shown in red is the uncertainty band extracted from the joint fit to all four channels simultaneously.

compared to the MINOS low- $Q^{2}$ correction in Fig. 12. Our fits obtain a suppression factor that is similar to the MINOS one, with almost identical suppression at $Q^{2}=0$, albeit with less curvature, particularly in the $\nu_{\mu} \mathrm{CC} 1 \pi^{ \pm}$and $\nu_{\mu} \mathrm{CCN} \pi^{ \pm}$channels. The correction factors from the fit with FrInel or FrAbs as free parameters give similar results.

The correlation matrices for the fits including a $Q^{2}$-dependent suppression are provided in Fig. 13. Again, the ANL/BNL input prior covariance is maintained. The parameters largely correlate in the same way for the FrAbs and FrInel fit, and for the FrInel fit the $R_{1}$ and $R_{2}$ parameters are negatively correlated.

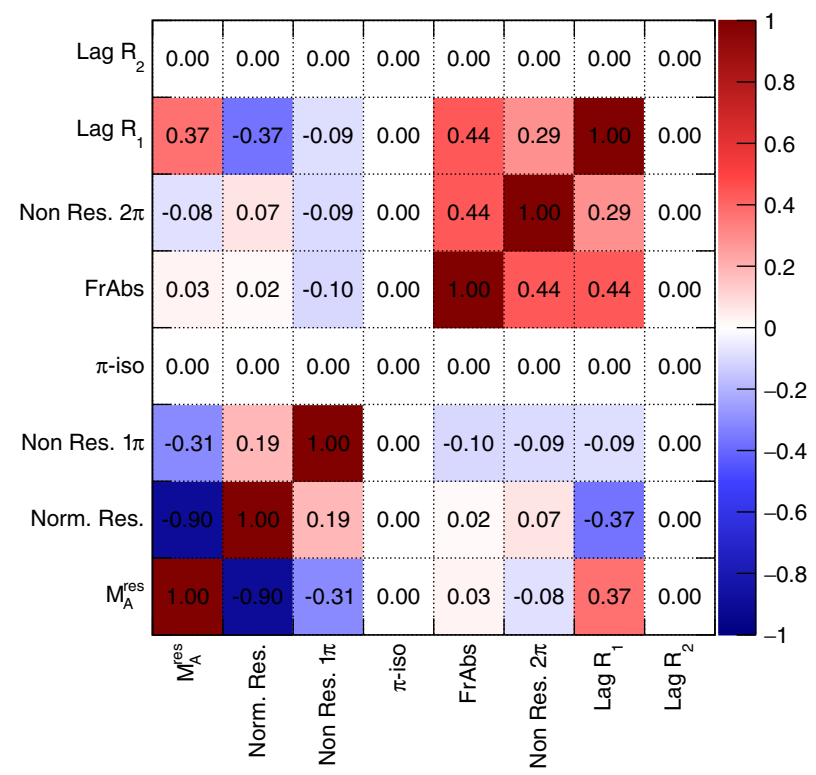

Figure 14 (Fig. 15) shows the ratio of the resulting fits to the MINER $\nu$ A data when FrAbs (FrInel) is taken as a free parameter. As anticipated, the predictions now have better agreement with the data in regard to the $\theta_{\mu}$ distribution, and the $\chi^{2}$ values are improved by the introduction of our ad hoc low- $Q^{2}$ correction. Other fit parameters are for the most part unchanged by the introduction of the low- $Q^{2}$ correction. Furthermore, $M_{\mathrm{A}}^{\text {res }}$ and NormRes are closer to their values when fitting ANL and BNL data, indicating the $Q^{2}$ correction alleviates the tension between nucleon and nuclear modeling. Figure 11 shows the comparison of all our models directly against MINER $\nu \mathrm{A}$ data in $Q^{2}$.

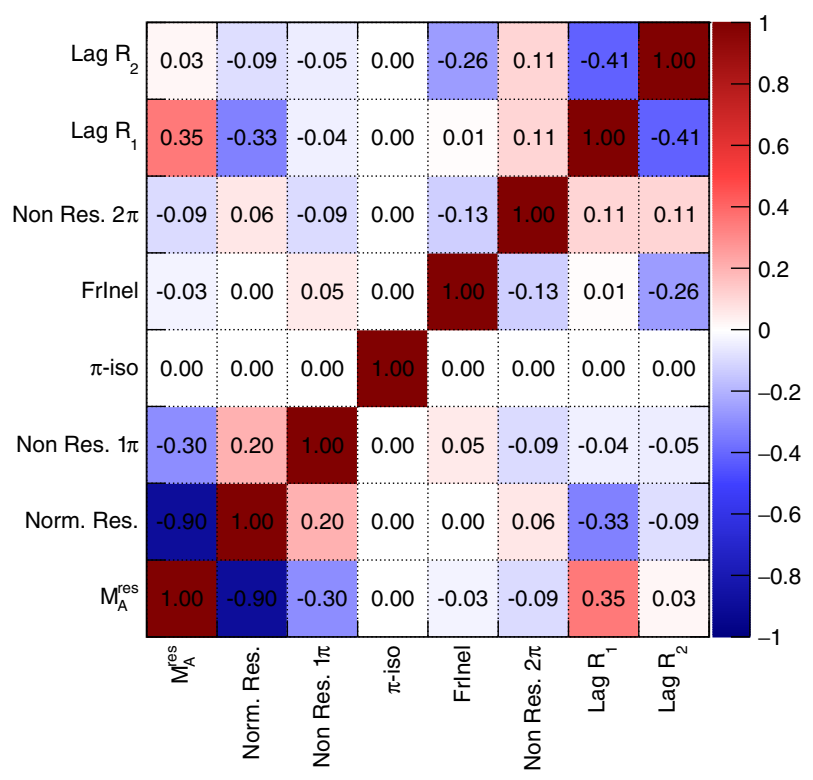

FIG. 13. Correlation matrix from tuning GENIE parameters with an ad hoc low- $Q^{2}$ supression with FrAbs included as a fit parameter (left panel) and with FrInel included as a fit parameter (right panel). 

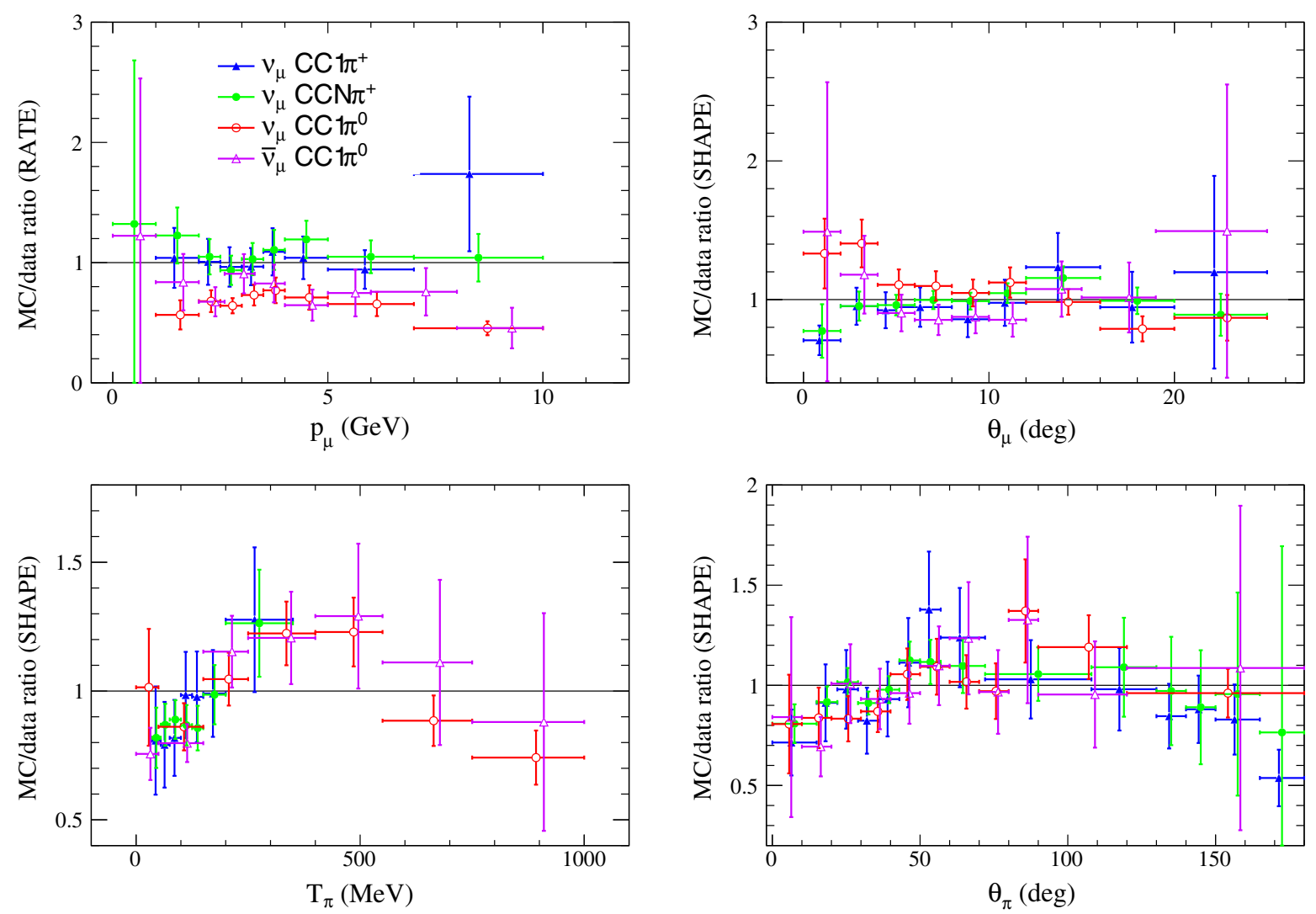

FIG. 14. MC/data ratios at the best-fit points from the FrAbs tuning with low- $Q^{2}$ suppression included.
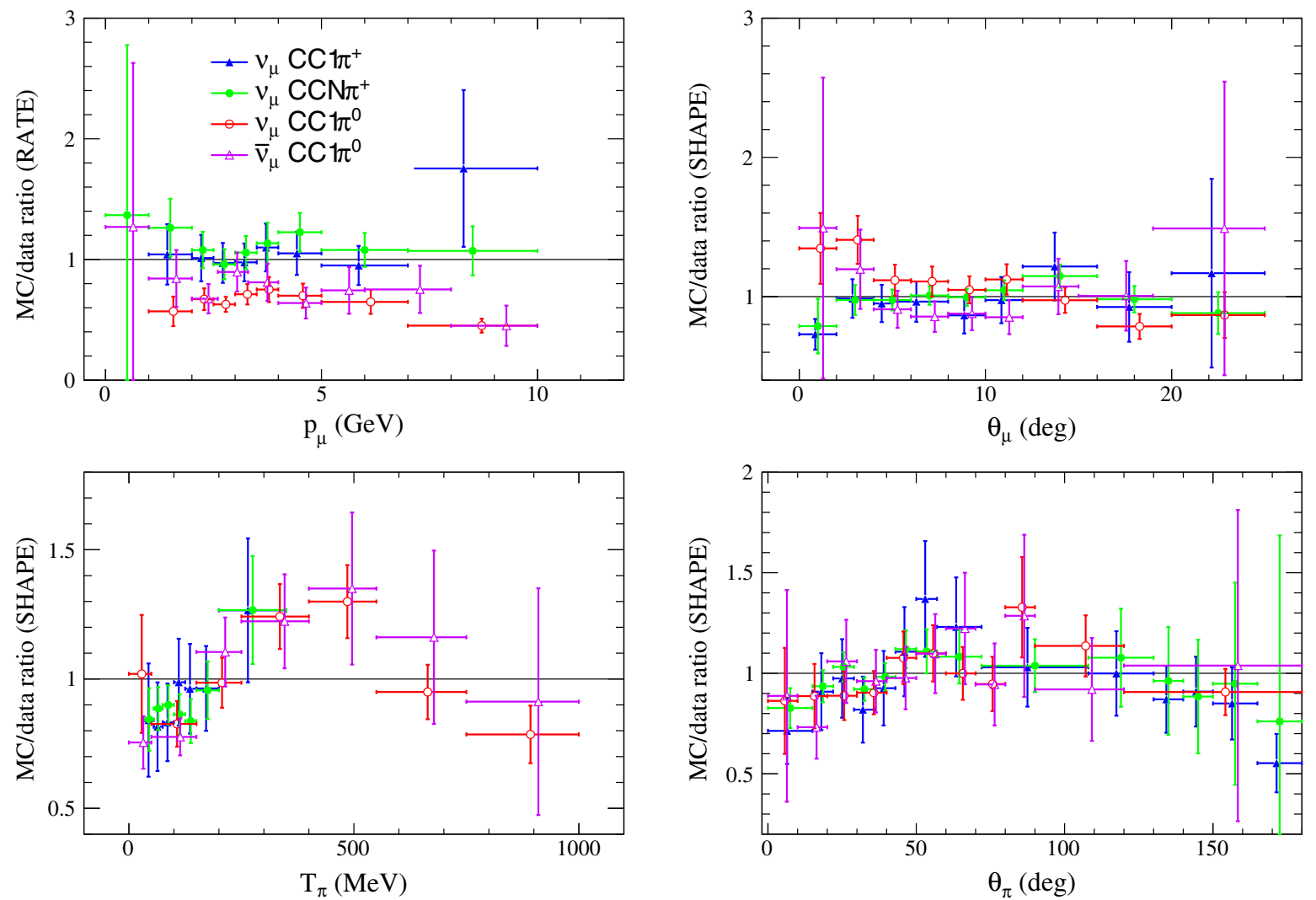

FIG. 15. MC/data ratios at the best-fit points from the FrInel tuning with low- $Q^{2}$ suppression included. 
TABLE IX. Individual channel FrAbs + low- $Q^{2}$ tuning results.

\begin{tabular}{lcccc}
\hline \hline Parameter & $\nu_{\mu} \mathrm{CC} 1 \pi^{ \pm}$ & $\nu_{\mu} \mathrm{CCN} \pi^{ \pm}$ & $\nu_{\mu} \mathrm{CC} 1 \pi^{0}$ & $\bar{\nu}_{\mu} \mathrm{CC} 1 \pi^{0}$ \\
\hline$M_{\mathrm{A}}^{\text {res }}(\mathrm{GeV})$ & $0.93 \pm 0.02$ & $0.92 \pm 0.02$ & $0.96 \pm 0.05$ & $0.94 \pm 0.05$ \\
NormRes $(\%)$ & $115 \pm 3$ & $117 \pm 3$ & $114 \pm 7$ & $115 \pm 7$ \\
NonRes $\pi(\%)$ & $43 \pm 4$ & $43 \pm 4$ & $45 \pm 4$ & $43 \pm 4$ \\
NonRes $2 \pi(\%)$ & 300 (limit) & $70 \pm 28$ & 300 (limit) & 300 (limit) \\
$\pi$-iso & $1=$ Iso (limit) & $1=$ Iso (limit) & $1=$ Iso (limit) & $1=$ Iso (limit) \\
FrAbs $(\%)$ & $92 \pm 65$ & $79 \pm 40$ & $74 \pm 22$ & $34 \pm 35$ \\
Lag. $R_{1}$ & $0.53 \pm 0.16$ & $0.43 \pm 0.13$ & $0.21 \pm 0.14$ & $0.14 \pm 0.22$ \\
Lag. $R_{2}$ & 0.50 (limit) & 0.50 (limit) & $0.63 \pm 0.31$ & 1.00 (limit) \\
MINER $\nu \mathrm{A} \chi^{2}$ & 32.2 & 55.7 & 71.2 & 27.7 \\
$\chi_{\text {pen }}^{2}$ & 0.1 & 0.4 & 0.5 & 0.0 \\
Total $\chi^{2}$ & 32.3 & 56.1 & 71.7 & 30 \\
$N_{\text {DoF }}$ & 33 & 34 & & 31 \\
\hline
\end{tabular}

TABLE X. Individual channel FrInel + low- $Q^{2}$ tune tuning results.

\begin{tabular}{lcccc}
\hline \hline Parameter & $\nu_{\mu} \mathrm{CC} 1 \pi^{ \pm}$ & $\nu_{\mu} \mathrm{CCN} \pi^{ \pm}$ & $\nu_{\mu} \mathrm{CC} 1 \pi^{0}$ & $\bar{\nu}_{\mu} \mathrm{CC} 1 \pi^{0}$ \\
\hline$M_{\mathrm{A}}^{\text {res }}(\mathrm{GeV})$ & $0.93 \pm 0.02$ & $0.91 \pm 0.02$ & $0.95 \pm 0.05$ & $0.94 \pm 0.05$ \\
NormRes $(\%)$ & $116 \pm 3$ & $117 \pm 3$ & $114 \pm 7$ & $115 \pm 7$ \\
NonRes $\pi(\%)$ & $43 \pm 4$ & $43 \pm 4$ & $44 \pm 4$ & $43 \pm 4$ \\
NonRes $2 \pi(\%)$ & 300 (limit) & $78 \pm 28$ & 300 (limit) & 300 (limit) \\
$\pi$-iso & $1=$ Iso (limit) & $1=$ Iso (limit) & $1=$ Iso (limit) & $1=$ Iso (limit) \\
FrInel $(\%)$ & $179 \pm 63$ & $173 \pm 37$ & $8 \pm 125$ & $103 \pm 57$ \\
Lag. $R_{1}$ & $0.49 \pm 0.14$ & $0.38 \pm 0.13$ & $0.25 \pm 0.17$ & $0.31 \pm 0.26$ \\
Lag. $R_{2}$ & 0.50 (limit) & 0.50 (limit) & $0.76 \pm 0.37$ & 1.00 (limit) \\
MINER $\nu \mathrm{A} \chi^{2}$ & 30.8 & 52.1 & 69.5 & 30.9 \\
$\chi_{\text {pen }}^{2}$ & 0.1 & 0.6 & 0.2 & 0.0 \\
Total $\chi^{2}$ & 30.9 & 52.7 & 69.7 & 30 \\
$N_{\text {DoF }}$ & 33 & 34 & & 30.9 \\
\hline \hline
\end{tabular}

TABLE XI. Channel-by-channel contributions to the $\chi^{2}$ at for the GENIE tunings with and without the low- $Q^{2}$ correction included.

\begin{tabular}{lcccccc}
\hline \hline Distribution & Channel & $N_{\text {bins }}$ & FrAbs tune & FrAbs + low- $Q^{2}$ tune & FrInel tune & FrInel + low- $Q^{2}$ tune \\
\hline$p_{\mu}$ (rate) & $\nu_{\mu} \mathrm{CC} 1 \pi^{ \pm}$ & 8 & 12.0 & 10.8 & 12.3 & 10.9 \\
& $\nu_{\mu} \mathrm{CC} N \pi^{ \pm}$ & 9 & 26.1 & 16.2 & 26.8 & 17.9 \\
& $\nu_{\mu} \mathrm{CC} 1 \pi^{0}$ & 8 & 19.0 & 26.2 & 19.3 & 26.9 \\
& $\bar{\nu}_{\mu} \mathrm{CC} 1 \pi^{0}$ & 9 & 6.2 & 7.1 & 6.3 & 7.2 \\
$\theta_{\mu}$ (shape) & $\nu_{\mu} \mathrm{CC} 1 \pi^{ \pm}$ & 9 & 7.5 & 7.4 & 7.4 & 7.1 \\
& $\nu_{\mu} \mathrm{CC} N \pi^{ \pm}$ & 9 & 4.0 & 6.3 & 4.1 & 5.6 \\
& $\nu_{\mu} \mathrm{CC} 1 \pi^{0}$ & 9 & 44.5 & 20.0 & 10.3 & 20.5 \\
$T_{\pi}$ (shape) & $\bar{\nu}_{\mu} \mathrm{CC} 1 \pi^{0}$ & 9 & 10.2 & 7.0 & 2.3 & 2.9 \\
& $\nu_{\mu} \mathrm{CC} 1 \pi^{ \pm}$ & 7 & 2.5 & 2.5 & 29.4 & 2.4 \\
& $\nu_{\mu} \mathrm{CC} N \pi^{ \pm}$ & 7 & 31.2 & 28.9 & 29.9 & 32.0 \\
& $\nu_{\mu} \mathrm{CC} 1 \pi^{0}$ & 7 & 30.9 & 16.1 & 16.0 & 18.7 \\
\hline
\end{tabular}


TABLE XI. (Continued)

\begin{tabular}{lcccccc}
\hline \hline Distribution & Channel & $N_{\text {bins }}$ & FrAbs tune & FrAbs + low- $Q^{2}$ tune & FrInel tune & FrInel + low- $Q^{2}$ tune \\
\hline$\theta_{\pi}$ (shape) & $\nu_{\mu} \mathrm{CC} 1 \pi^{ \pm}$ & 14 & 13.0 & 13.4 & 12.6 & 12.6 \\
& $\nu_{\mu} \mathrm{CC} N \pi^{ \pm}$ & 14 & 6.9 & 7.0 & 6.2 & 6.3 \\
& $\nu_{\mu} \mathrm{CC} 1 \pi^{0}$ & 11 & 8.3 & 12.2 & 8.9 & 9.4 \\
& $\bar{\nu}_{\mu} \mathrm{CC} 1 \pi^{0}$ & 11 & 3.4 & 4.4 & 3.5 & 3.7 \\
Total $\chi^{2}$ & & 148 & 242.3 & 212.2 & 240.7 & 215.7 \\
\hline \hline
\end{tabular}

Although the tuning sees improvement in the $\chi^{2}$ for the $\nu_{\mu} \mathrm{CC} 1 \pi^{0}$ and $\bar{\nu}_{\mu} \mathrm{CC} 1 \pi^{0}$ distributions, the $\nu_{\mu} \mathrm{CC} 1 \pi^{ \pm}$and $\nu_{\mu} \mathrm{CCN} \pi^{ \pm}$distributions get worse, hinting at tensions in the charged and neutral pion production channels.

Tables IX and X show the results of the fits to individual channels, and Table XI shows the breakdown of contributions to the $\chi^{2}$ from the individual channels. The best-fit $\chi^{2}$ value was significantly improved for each channel tuning when using a low- $Q^{2}$ suppression, and the extracted parameters were consistent with the ANL/BNL tunings. Pion kinematic distributions are not improved, and in some cases are slightly worse, as a result of including the low- $Q^{2}$ suppression. It is clear from Table VIII (or by comparing Tables VI and IX) that the low- $Q^{2}$ suppression has a similar effect in the fit to the FrAbs parameter. When the low- $Q^{2}$ suppression is introduced, FrAbs tends to consistently lower values. It is also clear that the $\bar{\nu}_{\mu} \mathrm{CC} 1 \pi^{0}$ channel favors stronger low- $Q^{2}$ suppression than the other channels.

\section{CONCLUDING REMARKS}

We have adjusted the parameters of the GENIE model that are important for pion production to match MINER $\nu \mathrm{A}$ data in the $\nu_{\mu} \mathrm{CC} 1 \pi^{ \pm}, \nu_{\mu} \mathrm{CC} N \pi^{ \pm}, \nu_{\mu} \mathrm{CC} 1 \pi^{0}$, and $\bar{\nu}_{\mu} \mathrm{CC} 1 \pi^{0}$ channels, using the NUISANCE framework. We incorporate existing results, which informs the GENIE model using ANL and BNL bubble chamber data from scattering off protons and deuterons. Fits of selected GENIE model parameters were done using the kinematic distributions $p_{\mu}, \theta_{\mu}, T_{\pi}$, and $\theta_{\pi}$. Parameter fits were performed with either the fraction of pions absorbed or the fraction of pions inelastically scattered in FSIs as a floating parameter, with broadly similar conclusions for the two cases.

The results of the fit (see Table V) show that the tuning improves the GENIE pion production model significantly, but tensions remain. The pull on the ANL/BNL prior demonstrates a tension between MINER $\nu \mathrm{A}$ nuclear target data and the light-target bubble chamber datasets used to make the prior, indicating a deficiency in the GENIE nuclear model which cannot be fixed by modifying the available reweighting dials. Additionally, fitting to individual MINER $\nu \mathrm{A}$ pion production channels produces different best-fit parameters, demonstrating that GENIE cannot describe the different exclusive channels in a consistent manner with the available dials (shown in Tables VI and
VII). Because the four channels cover different kinematic regions (see Table I) and contain different physics (e.g., different coherent pion production contributions or nonresonant processes), it is difficult to pinpoint the origin of the discrepancy between the model and the different MINER $\nu \mathrm{A}$ datasets.

Following experimental hints of discrepancies at low $Q^{2}$ for a variety of cross-section measurements on nuclear targets, an additional empirical low- $Q^{2}$ suppression was introduced and the fits were repeated. Although the data showed a preference for a strong suppression at low- $Q^{2}$ and the agreement improved for $\theta_{\mu}$ and $Q^{2}$ distributions, tensions remain. In particular, fits to individual MINER $\nu \mathrm{A}$ channels still produced different results, and favor different parameter values for the low- $Q^{2}$ suppression.

The main conclusion of this work is that current neutrino experiments operating in the few- $\mathrm{GeV}$ region should think critically about single pion production models and uncertainties, as the Monte Carlo models which are currently widely used in the field are unable to explain multiple datasets, even when they are from a single experiment.

A key strength of this analysis is its development within the NUISANCE framework, allowing it to be easily repeated with alternate model assumptions, neutrino interaction generators, and different data. The developments presented here will be used in future iterations of this work, as the MINER $\nu \mathrm{A}$ Collaboration works towards a GENIE model that provides a good description of all their available data, and can be easily applied to other measurements and experiments.

\section{ACKNOWLEDGMENTS}

P. S., L.P., and C. Wret would like to thank the UK Science and Technology Facilities Council (STFC) for Ph.D. funding support. P. S. acknowledges the Fermilab Neutrino Physics Center for the scholarship that funded this work and thanks Fermilab and the MINER $\nu$ A Collaboration for their hospitality during this work. C. Wilkenson acknowledges the support of the Swiss National Science Foundation and SERI. This document was prepared by members of the MINER $\nu \mathrm{A}$ Collaboration using the resources of the Fermi National Accelerator Laboratory (Fermilab), a U.S. Department of Energy, Office of Science, HEP User Facility. Fermilab is managed by Fermi Research Alliance, LLC (FRA), acting 
under Contract No. DE-AC02-07CH11359. These resources included support for the MINER $\nu \mathrm{A}$ construction project, and support for construction also was granted by the U.S. National Science Foundation under Grant No. PHY-0619727 and by the University of Rochester. Support for participating scientists was provided by NSF and DOE (USA); by CAPES and $\mathrm{CNPq}$ (Brazil); by CoNaCyT (Mexico); by Proyecto Basal FB 0821, CONICYT PIA ACT1413, Fondecyt 3170845, and 11130133 (Chile); by CONCYTEC, Dirección de Gestión de la Investigación-Pontificia Universidad Católica del Perú (DGI-PUCP), and Vicerrectorado de InvestigaciónUniversidad Nacional de Ingeniería (VRI-UNI) (Peru); and by the Latin American Center for Physics (CLAF); NCN Opus Grant No. 2016/21/B/ST2/01092 (Poland). We thank the MINOS Collaboration for use of its near detector data. Finally, we thank the staff of Fermilab for support of the beam line, the detector, and the computing infrastructure.
[1] U. Mosel and K. Gallmeister, Phys. Rev. C 96, 015503 (2017).

[2] L. Alvarez-Ruso et al., Prog. Part. Nucl. Phys. 100, 1 (2018).

[3] R. González-Jiménez, K. Niewczas, and N. Jachowicz, Phys. Rev. D 97, 013004 (2018).

[4] C. Wilkinson et al., Phys. Rev. D 93, 072010 (2016).

[5] K. Mahn, C. Marshall, and C. Wilkinson, Annu. Rev. Nucl. Part. Sci. 68, 105 (2018).

[6] P. Stowell et al., J. Instrum. 12, P01016 (2017).

[7] C. Andreopoulos et al., Nucl. Instrum. Methods Phys. Res., Sect. A 614, 87 (2010).

[8] C. Andreopoulos et al., arXiv:1510.05494.

[9] M. Kabirnezhad, Phys. Rev. D 97, 013002 (2018).

[10] T. Leitner, O. Buss, L. Alvarez-Ruso, and U. Mosel, Phys. Rev. C 79, 034601 (2009).

[11] J. Nieves, I. R. Simo, and M. J. V. Vacas, Phys. Rev. C 83, 045501 (2011).

[12] E. Hernandez and J. Nieves, Phys. Rev. D 95, 053007 (2017).

[13] P. Rodrigues, C. Wilkinson, and K. McFarland, Eur. Phys. J. C 76, 474 (2016).

[14] C. Wilkinson, P. Rodrigues, S. Cartwright, L. Thompson, and K. McFarland, Phys. Rev. D 90, 112017 (2014).

[15] P. Adamson et al. (MINOS Collaboration), Phys. Rev. D 91, 012005 (2015).

[16] A. A. Aguilar-Arevalo et al. (MiniBooNE Collaboration), Phys. Rev. D 83, 052007 (2011).

[17] B. Eberly et al. (MINER $\nu$ A Collaboration), Phys. Rev. D 92, 092008 (2015).

[18] C. L. McGivern et al. (MINER $\nu$ A Collaboration), Phys. Rev. D 94, 052005 (2016).

[19] O. Altinok et al. (MINER $\nu$ A Collaboration), Phys. Rev. D 96, 072003 (2017), Erratum to be submitted.

[20] L. Aliaga et al. (MINER $\nu$ A Collaboration), Nucl. Instrum. Methods Phys. Res., Sect. A 743, 130 (2014).

[21] T. Katori, Ph.D. thesis, University of Indiana, 2008.

[22] R. A. Smith and E. J. Moniz, Nucl. Phys. B43, 605 (1972).

[23] A. Bodek and J. Ritchie, Phys. Rev. D 23, 1070 (1981).

[24] J. Nieves, J. E. Amaro, and M. Valverde, Phys. Rev. C 70, 055503 (2004).

[25] R. Gran, J. Nieves, F. Sanchez, and M. J. V. Vacas, Phys. Rev. D 88, 113007 (2013).
[26] C. E. Patrick et al. (MINER $\nu$ A Collaboration), Phys. Rev. D 97, 052002 (2018).

[27] A. Mislivec et al. (MINER $\nu$ A Collaboration), Phys. Rev. D 97, 032014 (2018).

[28] L. Aliaga et al. (MINER $\nu$ A Collaboration), Phys. Rev. D 94, 092005 (2016).

[29] C. Andreopoulos et al. (GENIE Collaboration), Pregenerated public splines for GENIE 2.12.6, https://www .hepforge.org/archive/genie/data/2.12.6/ (2017).

[30] D. Rein and L. M. Sehgal, Ann. Phys. (N.Y.) 133, 79 (1981).

[31] K. M. Graczyk and J. T. Sobczyk, Phys. Rev. D 77, 053003 (2008).

[32] A. Bodek and U. K. Yang, J. Phys. G 29, 1899 (2003).

[33] T. Yang, C. Andreopoulos, H. Gallagher, K. Hoffmann, and P. Kehayias, Eur. Phys. J. C 63, 1 (2009).

[34] Z. Koba, H. B. Nielsen, and P. Olesen, Nucl. Phys. B40, 317 (1972).

[35] T. Sjöstrand, S. Mrenna, and P. Skands, Comput. Phys. Commun. 178, 852 (2008).

[36] D. Rein and L. M. Sehgal, Nucl. Phys. B223, 29 (1983).

[37] D. Rein and L. M. Sehgal, Phys. Lett. B 657, 207 (2007).

[38] C. Berger and L. M. Sehgal, Phys. Rev. D 79, 053003 (2009).

[39] S. Dytman, Acta Phys. Pol. B 40, 2445 (2009).

[40] R. A. Arndt, W. J. Briscoe, I. I. Strakovsky, and R. L. Workman, Phys. Rev. C 74, 045205 (2006).

[41] O. Buss, T. Gaitanos, K. Gallmeister, H. van Hees, M. Kaskulov, O. Lalakulich, A. B. Larionov, T. Leitner, J. Weil, and U. Mosel, Phys. Rep. 512, 1 (2012).

[42] G. M. Radecky et al. (ANL Collaboration), Phys. Rev. D 25, 1161 (1982).

[43] T. Kitagaki et al. (BNL Collaboration), Phys. Rev. D 34, 2554 (1986).

[44] S. J. Barish et al. (ANL Collaboration), Phys. Rev. D 19, 2521 (1979).

[45] T. Kitagaki et al. (BNL Collaboration), Phys. Rev. D 42, 1331 (1990).

[46] S. L. Adler, Ann. Phys. (N.Y.) 50, 189 (1968).

[47] P. Allen et al. (Aachen-Bonn-CERN-Munchen-Oxford Collaboration), Nucl. Phys. B176, 269 (1980).

[48] D. Allasia et al. (Amsterdam-Bologna-Padova-Pisa-SaclayTorino Collaboration), Z. Phys. C 20, 95 (1983).

[49] J. Bell et al., Phys. Rev. Lett. 41, 1012 (1978).

[50] F. James and M. Roos, Comput. Phys. Commun. 10, 343 (1975). 
[51] C. Berger and L. M. Sehgal, Phys. Rev. D 76, 113004 (2007).

[52] S. K. Singh, M. J. Vicente-Vacas, and E. Oset, Phys. Lett. B 416, 23 (1998).

[53] E. Oset and L. L. Salcedo, Nucl. Phys. A468, 631 (1987).

[54] S. X. Nakamura et al., Rep. Prog. Phys. 80, 056301 (2017).
[55] D. Casper, Nucl. Phys. B, Proc. Suppl. 112, 161 (2002).

[56] P. Przewlocki, Acta Phys. Pol. B 40, 2513 (2009).

[57] A. A. Aquilar-Arevalo et al. (MiniBooNE Collaboration), Phys. Rev. D 83, 052009 (2011).

[58] H. Gallagher, Nucl. Phys. B, Proc. Suppl. 112, 188 (2002). 